\title{
The mass of the neutron star in Vela $\mathrm{X}-\mathbf{1}^{\star}$
}

\author{
O. Barziv ${ }^{1,2}$, L. Kaper ${ }^{2}$, M. H. Van Kerkwijk ${ }^{3}$, J. H. Telting ${ }^{4}$, and J. Van Paradijs ${ }^{2,5, \star \star ~}$ \\ 1 European Southern Observatory, Karl-Schwarzschild-Strasse 2, 85748 Garching, Germany \\ 2 Astronomical Institute "Anton Pannekoek", University of Amsterdam and Center for High-Energy Astrophysics, \\ Kruislaan 403, 1098 SJ Amsterdam, The Netherlands \\ 3 Astronomical Institute, Utrecht University, PO Box 80000, 3508 TA Utrecht, The Netherlands \\ ${ }^{4}$ Isaac Newton Group of Telescopes, Netherlands Organisation for Scientific Research (NWO), Apartado 321, \\ 38700 Santa Cruz de La Palma, Spain \\ 5 Physics Department, University of Alabama, Huntsville, AL35899, USA
}

Received 19 February 2001 / Accepted 6 August 2001

\begin{abstract}
We measured the radial-velocity curve of HD 77581, the B-supergiant companion of the X-ray pulsar Vela X-1, using 183 high-resolution optical spectra obtained in a nine-month campaign. We derive radial-velocity amplitudes for different lines and wavelength regions, and find all are consistent with each other, as well as with values found in previous analyses. We show that one apparent exception, an anomalously low value derived from ultra-violet spectra obtained with the International Ultraviolet Explorer, was due to an error in the analysis procedures. We re-analyse all IUE spectra, and combine the resulting velocities with the ones derived from the new optical spectra presented here, as well as those derived from optical spectra published earlier. As in previous analyses, the radial velocities show strong deviations from those expected for a pure Keplerian orbit, with rootmean-square amplitudes of $\sim 7 \mathrm{~km} \mathrm{~s}^{-1}$ for strong lines of Si IV and $\mathrm{N}$ III near $4100 \AA$, and up to $\sim 20 \mathrm{~km} \mathrm{~s}^{-1}$ for weaker lines of N II and Al III near $5700 \AA$. The deviations likely are related to the pronounced line-profile variations seen in our spectra. Our hope was that the deviations would average out when a sufficient number of spectra were added together. It turns out, however, that systematic deviations as a function of orbital phase are present as well, at the $3 \mathrm{~km} \mathrm{~s}^{-1}$ level, with the largest deviations occurring near inferior conjunction of the neutron star and near the phase of maximum approaching velocity. While the former might be due to a photo-ionisation wake, for which we observe direct evidence in the profiles of $\mathrm{H} \delta$ and $\mathrm{H} \alpha$, the latter has no straightforward explanation. As a result, our best estimate of the radial-velocity amplitude, $K_{\mathrm{opt}}=21.7 \pm 1.6 \mathrm{~km} \mathrm{~s}^{-1}$, has an uncertainty not much reduced to that found in previous analyses, in which the influence of the systematic, phase-locked deviations had not been taken into account. Combining our velocity amplitude with the accurate orbital elements of the X-ray pulsar, we infer $M_{\mathrm{ns}} \sin ^{3} i=1.78 \pm 0.15 M_{\odot}{ }^{\star \star \star}$.
\end{abstract}

Key words. equation of state - stars: binaries: eclipsing - stars: early-type - stars: fundamental parameters stars: neutron - stars: pulsars: individual: Vela X-1

\section{Introduction}

Neutron stars follow a mass-radius relation that depends on the equation of state of the superdense matter in their interiors. For densities up to the nuclear saturation density $\left(\sim 10^{14.5} \mathrm{~g} \mathrm{~cm}^{-3}\right)$, the equation of state is reasonably well

Send offprint requests to: L. Kaper, e-mail: lexk@astro.uva.nl

* Based on observations collected at the European Southern Observatory, Chile (56.D-0251, 57.D-0409).

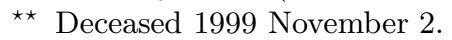

$\star \star \star$ The tables in the Appendix are only available in electronic form at the CDS via anonymous ftp to cdsarc.u-strasbg.fr $(130.79 .128 .5)$ or via http://cdsweb.u-strasbg.fr/cgi-bin/qcat?/A+A/377/925 constrained by laboratory measurements, but for higher densities it is still under much debate. For a given equation of state, there is a maximum possible mass of a neutron star. For a "soft" one (i.e., "compressible" matter), the maximum is near $1.5 M_{\odot}$, while for a "stiff" one (i.e., incompressible matter) it can be as high as $2 M_{\odot}$ (for recent reviews, see Heiselberg \& Pandharipande 2000; Lattimer \& Prakash 2000; Srinivasan 2001). In this respect, the $\mathrm{X}$-ray pulsar Vela X-1 is of particular interest, as it is the only neutron star for which there is strong evidence that it is more massive than the canonical $1.4 M_{\odot}$ (for a review, see Van Kerkwijk et al. 1995a; Thorsett \& Chakrabarthy 1999).

Vela X-1 (4U 0900-40) is a 283-s pulsar orbiting the B0.5 Ib supergiant HD 77581 in a period of 8.96 days. 
The X-ray source is powered by accretion from the B supergiant's wind (see Kaper 2001 for a recent overview of high-mass X-ray binaries hosting an OB-supergiant). Since the discovery of the X-ray pulsations in Vela X-1 by McClintock et al. (1976), its slightly eccentric $(e=$ 0.09 ) orbit has been determined with increasing accuracy from Doppler-shift measurements of pulse-arrival times. Recently, an update of the orbital parameters has been obtained based on the five-year long series of frequency measurements in hard X-ray flux with the Burst And Transient Source Experiment (BATSE) on board the Compton Gamma-Ray Observatory (Bildsten et al. 1997); see Table 4.

The evidence for Vela X-1's high mass is the relatively large amplitude, $K_{\mathrm{opt}}$, of the radial-velocity variations of the B supergiant companion, first noticed by Van Paradijs et al. (1976). From their analysis of close to a hundred photographic spectra, Van Paradijs et al. (1977a, hereafter Paper I) found $K_{\mathrm{opt}}=21.75 \pm 1.15 \mathrm{~km} \mathrm{~s}^{-1}$, which, combined with the BATSE orbit, corresponds to a neutronstar mass of $M_{\mathrm{ns}} \sin ^{3} i=1.79 \pm 0.11 M_{\odot}$ (here $M_{\mathrm{ns}}$ is the mass of the neutron star, and $i$ is the orbital inclination; uncertainties are $1 \sigma$ unless specified otherwise). Guided by the prospect that a more accurate mass determination of Vela X-1 might provide an important constraint on the equation of state appropriate for matter in neutron stars, Van Kerkwijk et al. (1995b, Paper II) made a detailed study of the radial-velocity curve of HD 77581, based on 40 high signal-to-noise CCD spectra, as well as 26 ultraviolet spectra obtained with the International Ultraviolet Explorer (IUE) and 13 nightly averages of digitized photographic spectra. They derived $K_{\text {opt }}=20.8 \pm 1.4 \mathrm{~km} \mathrm{~s}^{-1}$, confirming the value found in Paper I.

In Paper II, the accuracy of $K_{\mathrm{opt}}$ was found to be limited not by the statistical uncertainty in the radialvelocity measurements, but by systematic non-orbital contributions to the radial velocity. These contributions are correlated within a given night and can reach amplitudes up to $\sim 15 \mathrm{~km} \mathrm{~s}^{-1}$. They did not appear to be correlated with orbital phase. It was suggested that these velocity deviations might be caused by non-radial pulsations of HD 77581, similar to those seen in many early-type stars, perhaps excited by the varying tidal force excerted by the neutron star in its eccentric orbit. The situation may be complicated further by systematic effects on the spectra due to a photo-ionisation wake trailing the neutron star in its orbit (Kaper et al. 1994).

In Paper II, it was suggested that for a better understanding of these radial-velocity excursions, and thereby an improvement of the accuracy of the radial-velocity curve, either an extensive long-term sequence of spectroscopic observations would be required, with which one might "average out" the deviations, or a continuous spectroscopy campaign with a time resolution of a few hours, extending over at least one orbital cycle. We followed the first approach and monitored the binary system for a period of about 9 months, taking high-resolution spectra of HD 77581 on a night-by-night basis at the European Southern Observatory.

In Sect. 2, we describe the observations and the datareduction procedures. In Sect. 3, the spectra are presented. In Sects. 4 and 5, we describe the method of determining radial velocities and discuss the resulting radial-velocity curves. Section 6 is an intermezzo, dedicated to the reanalysis of IUE spectra, a subset of which was already used in Paper II in the determination of the mass; we compare our results to the ones found by Stickland et al. (1997) who analysed the same set of IUE data, and explain the origin of the difference between our results. In Sect. 7 , we combine all velocity curves. We also investigate systematic deviations with orbital phase, finding that, unfortunately, these are present and that they limit our final accuracy. In Sect. 8, we present our results for the mass of the neutron star and in Sect. 9 we discuss how these results influence the current status of our knowledge on the internal structure of neutron stars.

\section{Data reduction and analysis}

We observed HD 77581, the bright $(V=6.9 \mathrm{mag})$ B-supergiant companion to Vela X-1, in the period October 15, 1995, until July 15, 1996, using the 1.4-m Coudé Auxiliary Telescope (CAT) and Coudé Echelle Spectrograph (CES) at the European Southern Observatory (ESO), La Silla, Chile. The observations were carried out by the night assistant of the CAT (in La Silla or, more often, remotely from the ESO Headquarters in Garching, Germany) or by the astronomer present at the telescope.

Every night one spectrum (in a few cases two) of HD 77581 was obtained, as well as a few bias frames, two internal lamp flat fields and a Th-Ar wavelength calibration frame. Exposure times range from 20 to $30 \mathrm{~min}$ and the typical signal-to-noise ratio of the extracted spectra is $\gtrsim 300$ per $5 \mathrm{~km} \mathrm{~s}^{-1}$ resolution element. A fraction of the obtained spectra (47 out of 230) could not be used due to various practical problems (e.g., wavelength region covered by the stellar spectrum was not identical to that in the wavelength-calibration frame, no calibration frame available, wrong exposure level science frame, etc.).

The CES spectrograph has two "paths", one optimized for the blue and one for the red part of the optical spectrum. The CES long camera is equiped with one of two CCD detectors, CCD\#34 (2048 $\times 2048$ pixels $)$ or CCD\#38 $(2720 \times 520$ pixels, and thus a larger wavelength coverage $)$. In a given night, one has to select the red or the blue path, as well as the CCD. In order to minimize the impact of our programme on the observing run carried out during the remaining part of the night, we had to observe with the setting selected by the observer of that night. For this reason, we selected two wavelength regions, one in the blue (4077-4127 $\AA$ ) and one in the red path of the spectrograph (5670-5720 $\AA$ ). A total of 104 usable spectra were taken in the red and 79 in the blue wavelength region. A log 
of the observations is given in Tables A.1 and A.2 in the Appendix.

One of the main problems we were faced with during the analysis is the vignetting in the science frames, which varies from night to night, depending on the position of the telescope. It is caused by the small size of the field lens of the long camera (which was not designed for large CCDs), and the variations are due to the large distance between spectrograph and telescope. The CAT telescope is positioned in a dome just next to the dome of the $3.6 \mathrm{~m}$ telescope, while the CES spectrograph is located inside the $3.6 \mathrm{~m}$ dome. The light coming from one of the Nasmyth foci of the CAT goes through a pipe connecting the two domes, into the predisperser area of the spectrograph, bridging a distance of about $15 \mathrm{~m}$. For this reason, it is very difficult (especially in view of the regular earthquakes) to align the telescope and spectrograph beam. Dome flats provide a better way to correct for this misalignment than internal flats; unfortunately, in practice it was not feasible to obtain dome flats for our programme. The vignetting is more severe at longer wavelengths. The correction for the vignetting, though hampered by the relatively small wavelength region covered in one spectrum, has been carried out during the normalisation procedure.

Below, we describe the extraction, calibration, and normalisation of the spectra.

\subsection{Bias subtraction}

The electronic bias distribution is uniform over both chips, therefore in the bias correction (per night) we subtracted a number, derived either from the mean of the bias frames taken in that night (for the spectra taken from October 95 until March 96), or from the overscan of the science frame itself (for the spectra taken from March 96 until July 96).

\subsection{Flatfielding}

In order to apply the flatfield correction, we constructed one "master" flatfield frame for each of the two CCDs and the two wavelength regions, i.e., four frames in total. The master flatfield consists of the average of all available flatfields in a given wavelength region and taken with a given CCD, after inspection for saturation or under-exposure and a consistency check of their spectral parameters (central wavelength, resolution power, pre-disperser exit slit width). The individual flatfields were normalised to unity before averaging. Since the position of the spectrum on the CCD was not always the same during the extended period of our observing campaign, the master flatfield does not have a uniform exposure level. We scaled the flatfield to unity by dividing each row of the detector by its mean value. The night-to-night changes in instrument setting are small and have no significant effect on the flatfield structure (e.g., we were not confronted with strong detector fringes or ghost images).

\subsection{Optimal extraction}

We used an optimal extraction algorithm developed by Van Kerkwijk (1993), based on the method of Horne (1986), which includes corrections for small nonalignments of the spectrum with the dispersion direction of the chip, and detects and filters for cosmic-ray events. Some bad columns on the detectors have been corrected for by interpolation (in pixel space) over the gaps in the extracted spectrum.

In the red wavelength region some atmospheric water vapour lines are present. As they would cause problems in the velocity determination we removed them by linear interpolation.

\subsection{Wavelength calibration}

For the wavelength calibration we use the Th-Ar calibration frames, fitting the line positions with a second-order dispersion relation. The typical root-mean-square residuals are $0.001 \AA$. The dispersion is $0.018 \AA$ per pixel for the blue region and $0.024 \AA$ per pixel for the red region. There are about five pixels per resolution element.

\subsection{Barycentric correction and orbital ephemeris}

We corrected for the Earth's orbital and rotational motion by calculating the component of the Earth's velocity in the direction of HD 77581 at the mid-time of the observation and subtracting it from the velocity measured from the determined Doppler shift of the line (see Sect. 4). The orbital phase was calculated using the orbital parameters based on the BATSE data (Bildsten et al. 1997); these are listed in Table 4.

\subsection{Normalisation of the spectra}

Normalising the spectra was not a straightforward procedure, the main reason for this being the (time-dependent) vignetting (see above) which affects all spectra. We explored different methods to normalise the blue and the red spectra, taking into account: (i) the use of the same continuum regions (after shifting the spectra approximately to the reference frame of the star) for each spectrum and (ii) the assumption that the shape of the spectral continuum can be described by a polynomial of order four or lower.

The main problem we were confronted with was the lack of clean continuum regions due to the small wavelength region $(\sim 50 \AA)$ covered in each spectrum. Also, the intrinsic (i.e. non-orbital) variability of the stellar spectrum sometimes causes the lines to extend into the spectral continuum regions.

Below we describe the normalisation methods we preferred after testing different approaches. We note that we repeated the full analysis (including the cross-correlation procedure) for different normalisation methods, but did not find significantly different results. 


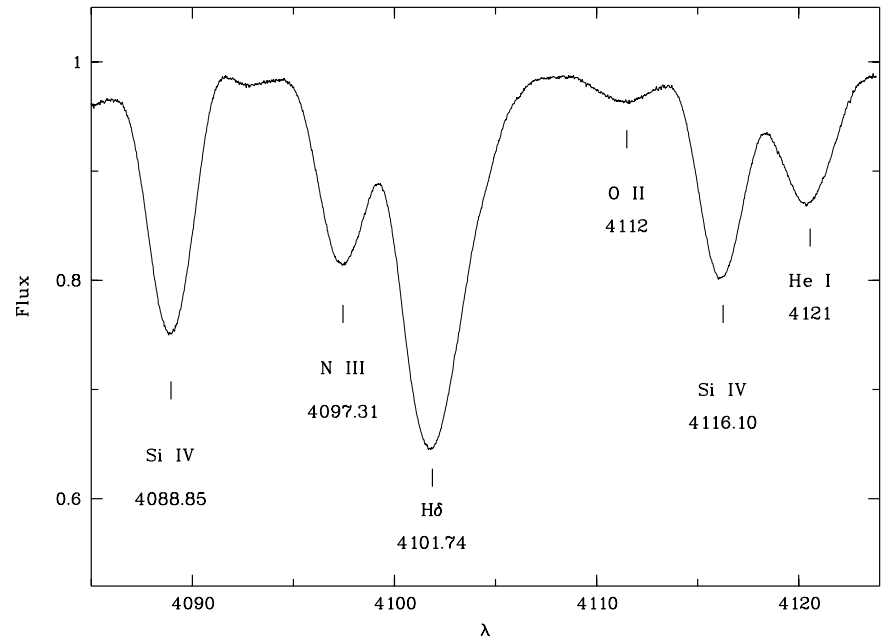

Fig. 1. The average blue CAT/CES spectrum centered at $\mathrm{H} \delta$ $4102 \AA$, with line identifications. The wavelength scale is in Ångstrøm.

Table 1. The wavelength regions used to normalise the spectra, for the blue and red wavelength region and CCD\#34 and 38, respectively. Before normalisation, the selected continuum regions are shifted by an amount corresponding to the (predicted) Doppler shift for the orbital phase at the time of observation.

\begin{tabular}{ll}
\hline CCD\#38 & CCD\#34 \\
\hline Blue & \\
$4080.45-4082.10$ & $4086.04-4086.50$ \\
$4091.25-4091.77$ & $4091.50-4092.50$ \\
$4107.10-4108.00$ & $4094.35-4094.95$ \\
$4122.62-4124.05$ & $4108.10-4108.50$ \\
& $4113.20-4113.70$ \\
& $4118.22-4118.77$ \\
Red & \\
$5672.30-5672.90$ & $5673.40-5673.55$ \\
$5682.82-5683.38$ & $5682.82-5683.38$ \\
$5693.00-5693.66$ & $5693.00-5693.66$ \\
$5699.98-5702.58$ & $5699.61-5700.38$ \\
$5714.25-5716.00$ & $5713.04-5714.04$ \\
\hline
\end{tabular}

\subsubsection{Blue spectra}

The central part of the blue spectrum (Fig. 1), i.e., at the blend of N III $4097 \AA$ and $\mathrm{H} \delta$, is not affected by vignetting, so the shape of the continuum is well reproduced by a linear function defined by the continuum regions left and right of the blend. The edges of the spectrum were normalised using two second-order polynomials, one at each side of the spectrum, which were forced to have the same slope as the linear function at the point where they meet. The normalisation regions are given in Table 1.

\subsubsection{Red spectra}

The effect of vignetting is more pronounced in the red wavelength region. Furthermore, the intrinsic variability of the line profiles is much larger compared to that in the blue

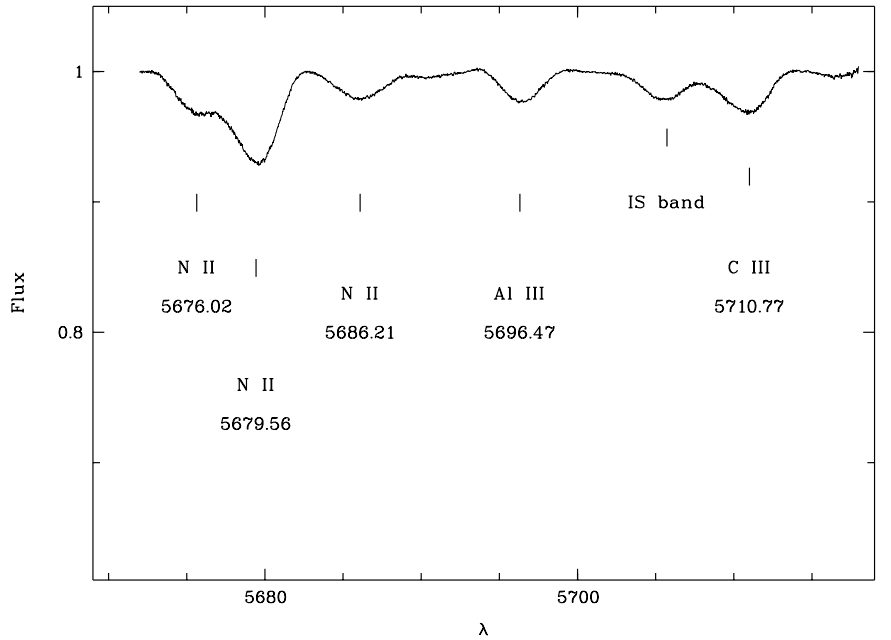

Fig. 2. The average red CAT/CES spectrum centered at $5695 \AA$, which includes several lines of a N II multiplet. Note the difference in strength between the lines present in the blue and red spectra.

spectrum, the spectral lines are closer to each other, and sometimes there appear to be (variable) P Cygni emission features at the blue side of the strongest $\mathrm{N}$ II lines. There are no extended continuum regions which are completely devoid from spectral features. The regions listed in Table 1 are those we believe are least affected by spectral lines.

In order to reduce the effect of the vignetting (which otherwise would force us to use a high-order polynomial to fit reasonably the shape of the continuum) we divided all the spectra by one spectrum (r8005r) which appears to be only moderately affected by vignetting. The residuals were fitted with a third-order polynomial. The latter fits should approximately reproduce the shape of the continuum and eliminate most of the vignetting. Then, we multiplied the original spectra with this fit. In this way, the spectra were "ironed". Finally, we fitted a fourth-order polynomial to the ironed spectra, using the normalisation regions listed in Table 1.

\section{Description of the spectra}

In Figs. 1 and 2 we show the average of the blue and of the red spectra, respectively, after having taken into account the difference in orbital motion. The blue spectrum includes stronger lines than the red spectrum, which makes the blue spectrum better suited to measure the star's radial velocity.

An overview of the blue and red spectra is presented in Figs. 3 and 6, respectively. The spectra are ordered according to orbital phase; the corresponding observing times can be found in Tables A.1 and A.2 in the Appendix. The few spectra obtained with CCD\#34 (instead of CCD\#38) are shown in Figs. 4 and 7. The wavelength scale of the spectra is with respect to the stellar rest frame, i.e., the orbital motion has been taken out to facilitate comparison. The figures show that the profiles of the lines in the 

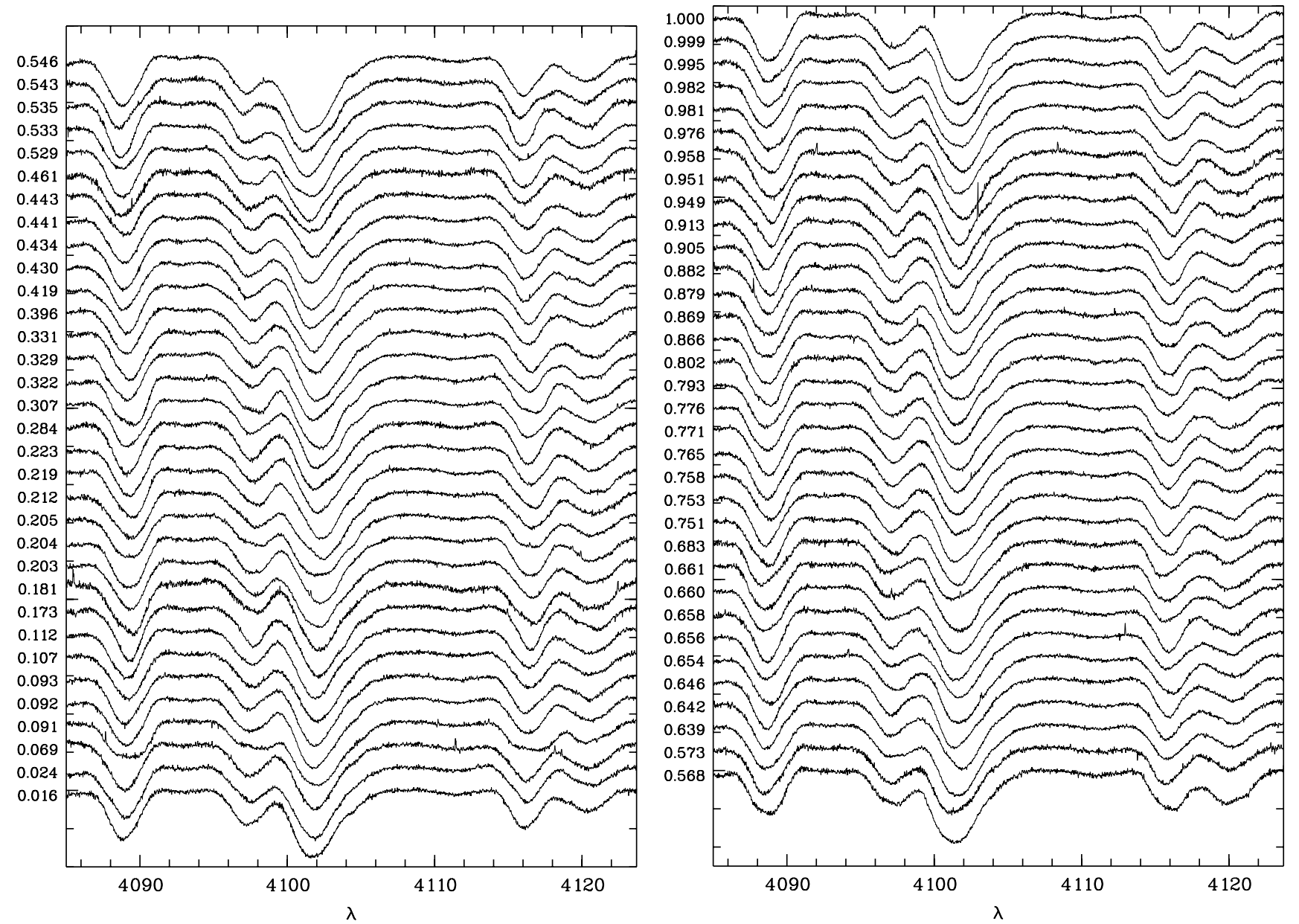

Fig. 3. The blue spectra obtained with CCD\#38 ordered according to orbital phase. The Doppler shift due to the orbital motion has been taken out. Note the variability of the line profiles, and the strong correlation in shape between the different lines. The $\mathrm{H} \delta$ line shows stronger variations in the blue wing when the X-ray source passes through the line of sight $(\phi=0.5)$. These variations are likely due to the presence of a photo-ionization wake in the B-supergiant's wind.

red spectra are much more variable than those of the lines in the blue spectra. In a given spectrum, the different lines seem to vary mostly in concert. In some cases the variations are dramatic, especially in the red spectra, where the depths of the photospheric lines are sometimes strongly reduced. The variations do not show an obvious dependence on orbital phase. In order to have a better view on possible orbital-phase related line-profile variations, we binned the spectra in nine phase bins (nine bins is chosen since the orbital period is very close to nine days). The results are shown in Figs. 5 and 8.

\subsection{The blue spectra centered on $H \delta 4102 \AA$}

The blue spectra cover $\mathrm{H} \delta$ and two strong Si IV lines, at 4088.85 and $4116.10 \AA$, as well as weaker lines of N III (at 4097.31 $\AA$ ), O II (4112 $\AA$ ) and He I (4121 $)$ ). Furthermore, there are two small features which we could not identify; these lines are also seen in the average spectrum in Paper II. These are located at $4084.88 \AA$ just shortward of the Si IV $4088.85 \AA$ line and at $4092.96 \AA$ at the opposite side of the Si IV line.

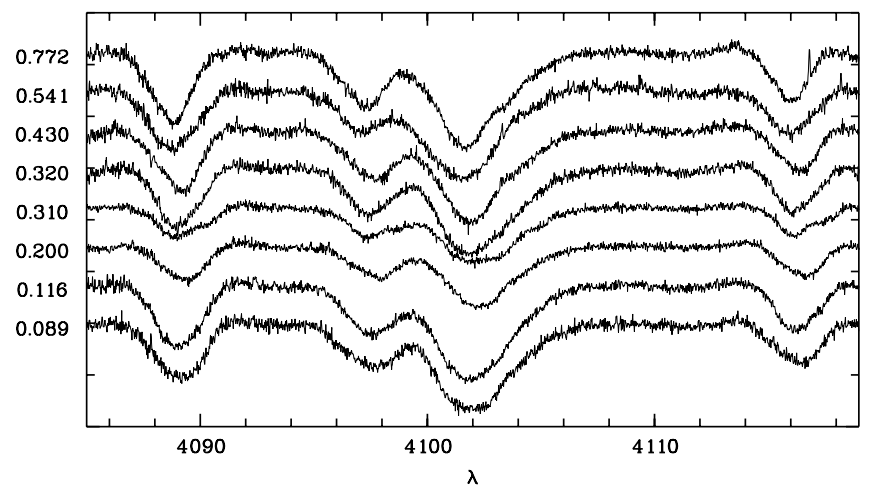

Fig. 4. The blue spectra obtained with CCD\#34, ordered according to orbital phase.

In general, the lines show strikingly similar behaviour. For the two Si IV lines this is expected, since they arise from the same multiplet. But the N III line at $4097.31 \AA$, while blended with the $\mathrm{H} \delta$ line, generally behaves similarly as well. See, e.g., the blue spectrum at phase 0.322 in Fig. 3, where the profiles of the Si IV lines and the N III line show an identical deformation of line shape. It is less 


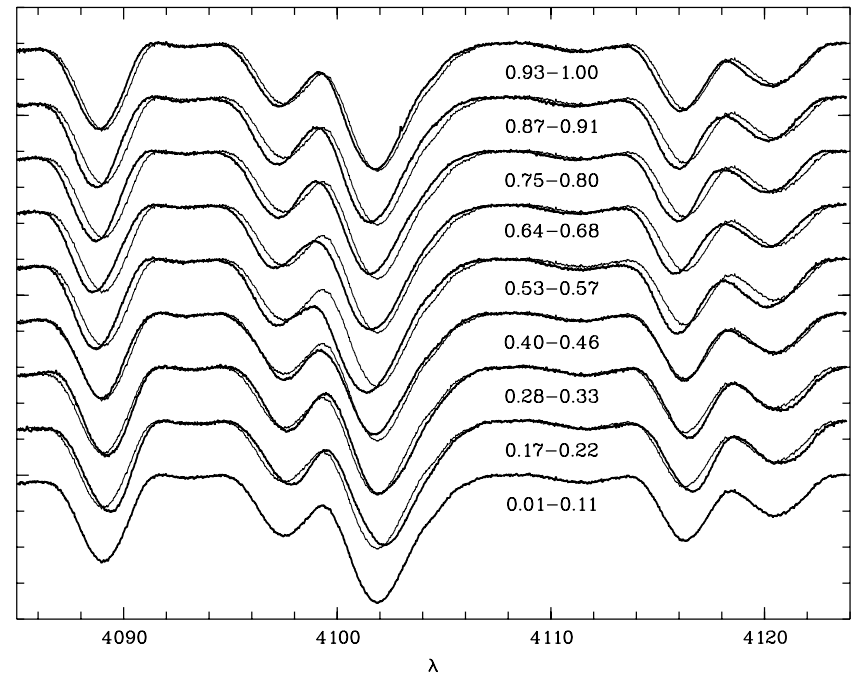

Fig. 5. Mean blue spectra, obtained after averaging over nine different phase bins. The thin line represents the mean profile at orbital phase 0.01-0.11 and is plotted for comparison.

easy to judge the case for the $\mathrm{O}$ II line, which is rather weak, and the He I line, which is not completely covered by our spectra (hence we did not include the latter in our analysis).

Also in the phase-binned spectra, the lines appear mostly to vary in concert. The profiles close to X-ray eclipse (in phase intervals $0.01-0.11$ and $0.95-1.00$ ) are rather symmetric, while the mean profiles in phase intervals $0.17-0.33$ and $0.75-0.80$ are shifted to the red, and those from $0.53-0.64$ appear asymmetric to the blue. The variations are quite a bit smaller, however, than the large internal spread within each phase interval.

The only line showing deviant behaviour is $\mathrm{H} \delta$. During orbital phases $0.45-0.65$, i.e., when the neutron star passes in front of the supergiant companion, the line profile is strongly distorted, especially the blue wing, where often a weak and blue-shifted absorption component can be observed, which can have such a high, blue-shifted velocity that it blends with the $\mathrm{N}$ III line. At other phases, the line profile behaviour is more similar to what is seen for the Si IV and N III lines.

In Fig. 5, the enhanced blue absorption in $\mathrm{H} \delta$ can be seen clearly in the mean profiles of phase intervals $0.40-$ 0.46 and $0.53-0.57$. This distortion of the $\mathrm{H} \delta$ line is very similar to that seen in the $\mathrm{H} \beta$ and He I $4471 \AA$ line of HD 77581 (Kaper et al. 1994), and is most likely caused by the perturbation of the B-supergiant wind due to the presence of the X-ray source (see Sect. 7.2).

\subsection{The red spectra centered at $5695 \AA$}

The red spectra cover lines which are much weaker than those in the blue, and which, as is clear from the spectra, also show much stronger intrinsic perturbations of their profiles. The lines covered include $\mathrm{N}$ II lines at 5676.02, 5679.56, and 5686.21 $\AA$, an $\mathrm{Al}$ III line at 5696.47 $\AA$, and an $\mathrm{C}$ III line at $5710.77 \AA$. Furthermore, the spectra contain a diffuse interstellar band at $5705 \AA$. Its constant profile demonstrates the stability of the instrument, but at the same time, since it is contaminating the $\mathrm{C}$ III line it hinders us from using the latter line to measure velocities. Finally, between the N II and the Al III line, at $\lambda 5691.10 \AA$, there is a very shallow variable absorption feature which we are not able to identify.

The N II lines belong to the same multiplet. All three lines show dramatic intrinsic variations, with, like for the blue spectra, little evidence for a correlation with orbital phase. On occasion, the distinction between the two blended N II lines disappears completely (see, e.g., phases 0.184 and 0.511 ), or an additional feature occurs (phases 0.337 or 0.458 ), in an apparently irregular manner. The strongest $\mathrm{N}$ II line sometimes appears to develop a red (P-Cygni) emission bump. If real, this would be an indication that the strongest $\mathrm{N}$ II line is formed in the outer expanding layers of the atmosphere. Note that the N II lines are often a useful diagnostic in studying non-radial pulsations in B-type stars (D.R. Gies, private communication). Figure 8 indicates that, on average, at orbital phases close to X-ray eclipse $(0.95-1,0-0.25)$ the red spectral lines are deepest.

In general, the $\mathrm{Al}$ III and $\mathrm{C}$ III line profiles are similar to those of the N II lines. See, e.g., the spectra at orbital phases 0.868 and 0.872 , where all the line profiles (except that of the interstellar band, of course) are distorted towards longer and shorter wavelengths, respectively. Again, at several orbital phases (e.g., 0.01) features appear which could be interpreted as being due to incipient emission. These can be present either on the left wing (Al III line, e.g., phases $0.01,0.296$, and 0.675 ), or on the right wing (Al III line, phase 0.448 ). In view of the difficulty of defining the continuum level, it is hard to say whether these are indeed emission features or whether there is a weak absorption feature right next to it.

Sometimes (see, e.g., the spectra obtained at phases 0.653 and 0.78 ) the depth of the lines decreases so much that the N II blend becomes very shallow, whereas the weaker N II and Al III lines almost disappear.

\section{Radial-velocity determination}

We determined the radial velocity of HD 77581 by means of cross-correlation. We used the same algorithm as in Paper II (see also Van Kerkwijk 1993) where each spectrum is cross-correlated with all other spectra in the data set. We decided to use all the available spectra as independent reference spectra, because of the lack of a template spectrum of a bright star with identical spectral type. We did not cross-correlate against the average spectrum to avoid auto-correlation effects which lead to a systematic decrease of the amplitude of the radial-velocity curve. For details on the cross-correlation method we refer to Paper II and Van Kerkwijk (1993).

We first transformed the spectra to a logarithmic wavelength scale so that a Doppler shift is a linear displacement along the spectrum. The top part of the cross-correlation 

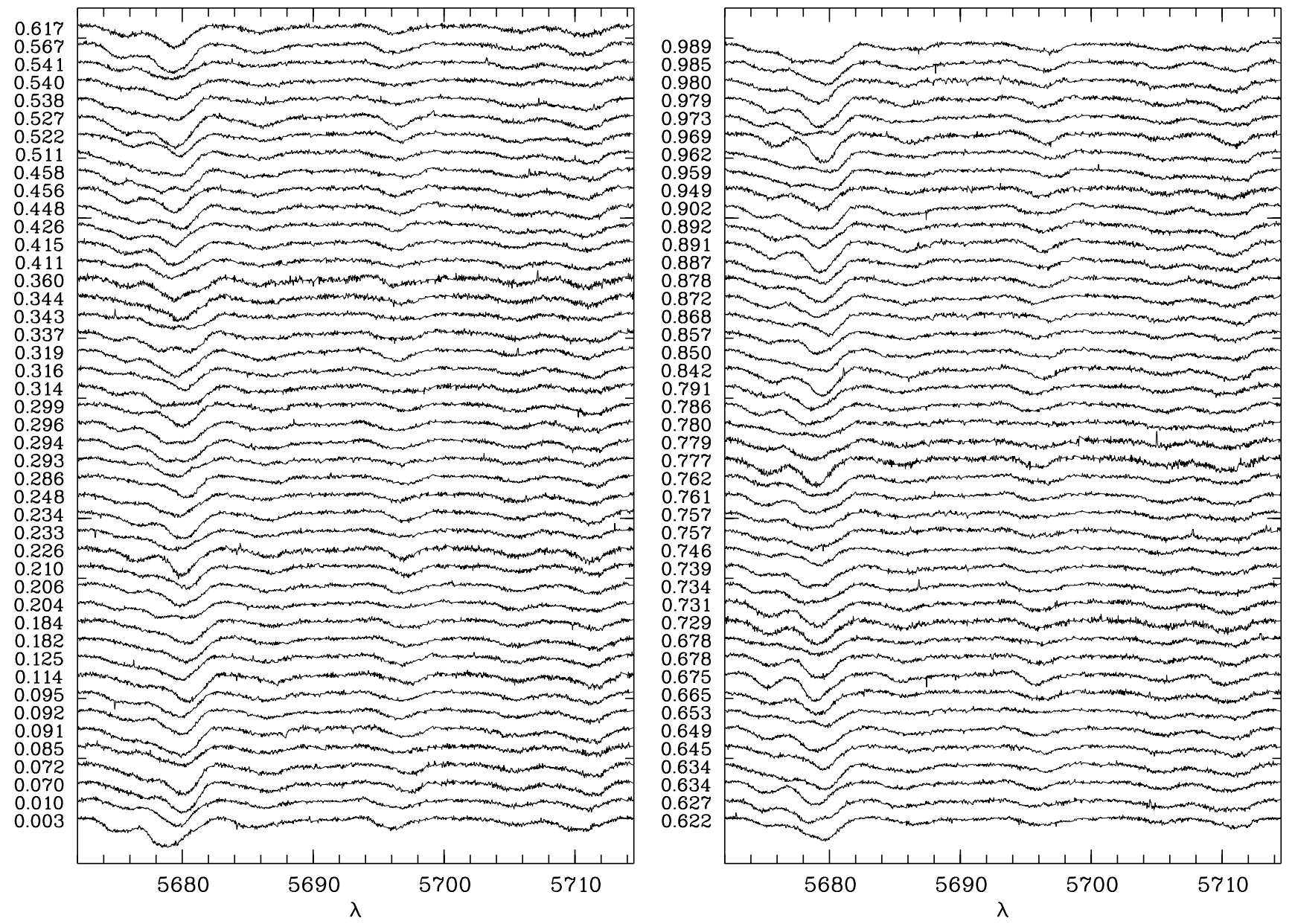

Fig. 6. The red spectra (CCD\#38) ordered according to orbital phase. The lines of the N II multiplet show strong intrinsic variability, apparently unrelated to orbital phase. In the most extreme cases, the lines almost disappear.

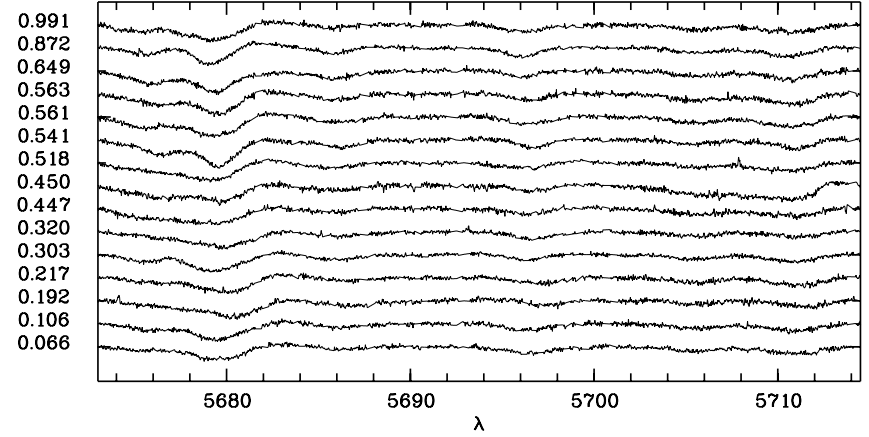

Fig. 7. The red spectra obtained with CCD\#34, ordered according to orbital phase.

function $(\mathrm{CCF})$ was fitted with an analytic function (a Gaussian plus a linear function) whose maximum determines the velocity shift of the spectrum. Obviously, the relevant part of the cross-correlation function is the area close to the maximum; we fit only the upper 30 to $40 \%$ of the CCF, depending on its shape and smoothness (Fig. 9).

We cross-correlated all the lines separately as well as combined, using the wavelength regions listed in Table 2. The measured radial velocities are listed in Table A.1 (blue wavelength region) and Table A.2 (red wavelength region)

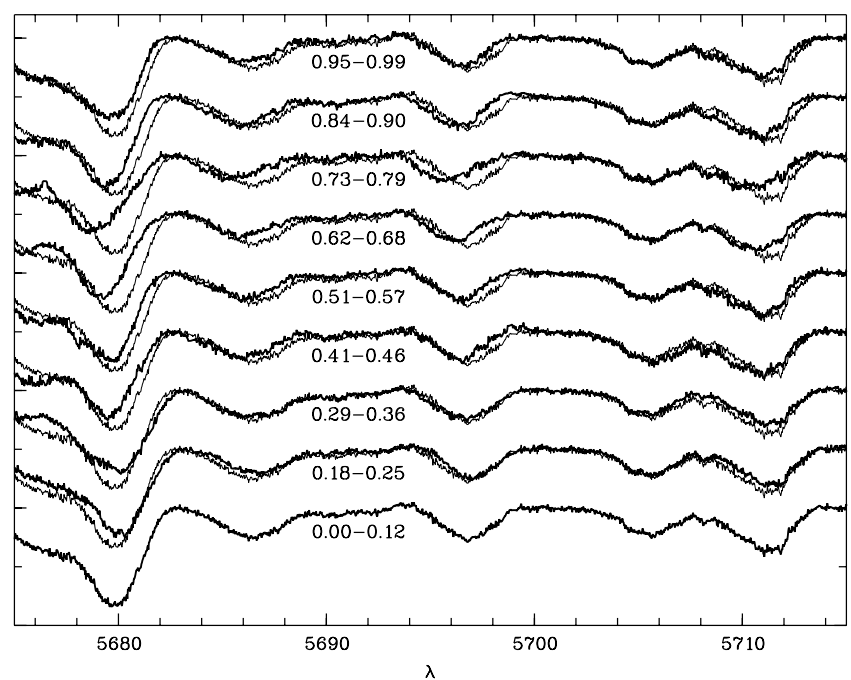

Fig. 8. Mean red spectra, obtained after averaging over nine different phase bins. The thin line represents the mean profile at orbital phase $0.01-0.11$ and is plotted for comparison.

in the Appendix. The typical intrinsic, formal accuracies are $0.7 \mathrm{~km} \mathrm{~s}^{-1}$ for the blue spectra and $1.5 \mathrm{~km} \mathrm{~s}^{-1}$ for the red spectra. 

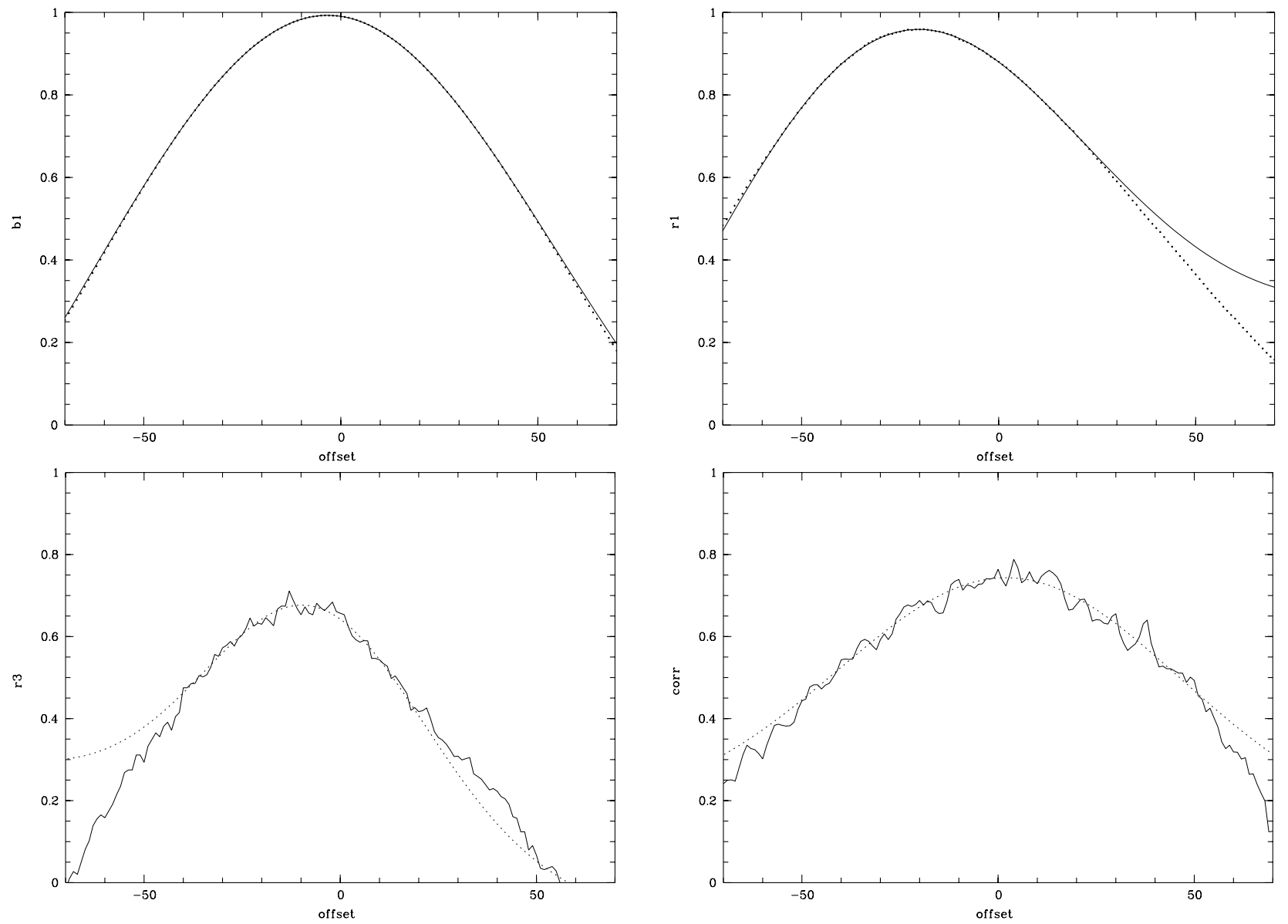

Fig. 9. A typical cross-correlation function and the fit applied to the area near the maximum for Si IV $4088.85 \AA$ (upper left), N II 5676/5679 $\AA$ blend (upper right), Al III 5696.47 $\AA$ (lower left) and the interstellar band (lower right). The offset is measured in $\mathrm{km} \mathrm{s}^{-1}$.

Table 2. Cross-correlation regions.

\begin{tabular}{ll}
\hline CCD\#38 & CCD\#34 \\
\hline Blue & \\
$4084.40-4092.45$ & $4085.00-4093.30$ \\
4092.50-4099.04 & $4093.30-4099.40$ \\
4108.30-4118.12 & $4110.13-4119.12$ \\
Red & \\
$5669.76-5682.98$ & $5673.20-5682.98$ \\
$5682.98-5693.78$ & $5682.98-5693.78$ \\
$5693.78-5701.91$ & $5693.78-5701.91$ \\
\hline
\end{tabular}

The cross-correlation method worked satisfactorily for the blue spectra. The CCF is very smooth and the fit well defined. This results in an accurate determination of the velocities. In Fig. 9, we show a typical CCF, derived from the cross-correlation of the Si IV line, as well as the fit.

In the red spectra, the CCF is much less well-defined, since the absorption features are much weaker. We partly overcame this by allowing the fit to the CCF to cover a larger area around the peak so that the determination of the peak position is not too much affected by the noise. Fortunately, the N II blend (at 5676.02 and $5679.56 \AA$ ), despite its strong intrinsic variations, is sufficiently deep to produce a reasonable CCF. In Fig. 9, we show a typical $\mathrm{CCF}$ of the $\mathrm{N}$ II blend and of the $\mathrm{Al}$ III line.

The presence of the interstellar line at $5705 \AA$ allows us to check for trends, e.g., due to instrumental effects, in the derived radial velocities. In the bottom-right panel in Fig. 9 we show the CCF for the diffuse interstellar band. It is rather wiggly, since the band is quite weak. Figure 10 displays the radial velocities derived from the band (filled circles, displaced by $-75 \mathrm{~km} \mathrm{~s}^{-1}$ ). For comparison, the radial-velocity curves of the N II $5686 \AA$ and $\mathrm{Al}$ III line regions are shown. The radial velocity of the DIB is consistent with a constant value $\left(\chi_{\text {red }}^{2}=1.1\right)$. A slight phase dependence might be introduced due to the neighbouring $\mathrm{C}$ III line, which moves close to the DIB around phase 0.75 , but this does not become apparent in Fig. 10. Nevertheless, to be on the safe side, we did not use the radial velocities measured from the $\mathrm{C}$ III line in our solutions.

From the above, we conclude that our error estimates are reasonable for the interstellar band. This feature does not vary in shape, and hence it satisfies the assumption underlying cross-correlation, viz., that the two spectra being correlated differ only in their radial velocity. This 


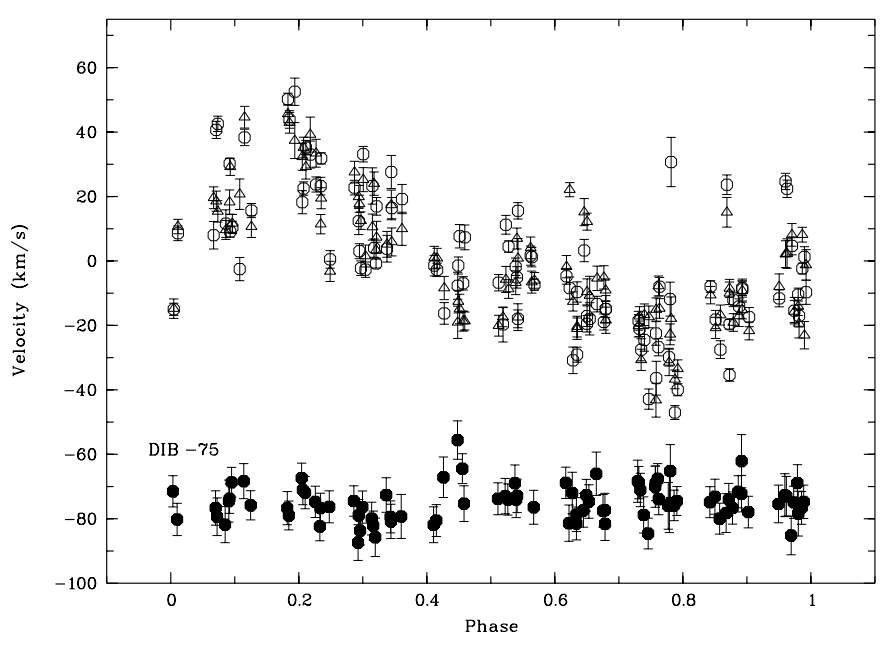

Fig. 10. The radial velocity of the diffuse interstellar band (DIB) as a function of orbital phase (filled circles, shifted by $-75 \mathrm{~km} \mathrm{~s}^{-1}$ ). For comparison, the radial velocities are shown for the $\mathrm{N}$ II $5686 \AA$ (triangles) and $\mathrm{Al}$ III (open circles) regions; these lines have comparable depth in the red spectra. As expected, the velocity of the DIB is consistent with a constant value.

assumption will not hold for the stellar lines, given the large variations seen in the line profiles. Therefore, the error estimates become somewhat ill-defined. Nevertheless, if all lines varied truly in concert, one would expect the inferred velocities to be the same within the errors.

In Fig. 11, we compare the radial velocities derived for the different lines. For the two regions centred on the Si IV lines, we find a good correspondence. However, while there are no systematic deviations, the velocities inferred for the lines are not consistent with each other within the formal uncertainties (root-mean-square difference of $2.1 \mathrm{~km} \mathrm{~s}^{-1}$; $\left.\chi_{\text {red }}^{2}=3.5\right)$, indicating that there are small differences. We do not believe that this could be due to differences in the behaviour of the two Si IV lines, which, as mentioned, arise from the same multiplet. Instead, it might be due to differences in other lines in the two regions; e.g., the $4116 \AA$ region is influenced by the wing of the He I $4121 \AA$ line.

Indeed, the velocities inferred from the $\mathrm{N}$ III region show larger differences with those inferred from the Si IV lines ( $\mathrm{rms}$ of $4.6 \mathrm{~km} \mathrm{~s}^{-1} ; \chi_{\text {red }}^{2}=10$ ), suggesting that the $\mathrm{N}$ III line profile varies in a somewhat different manner than the Si IV lines. This conclusion remains even if one removes the five most discrepant points, which occur near phase 0.5 , when the $\mathrm{N}$ III velocity may be biased towards positive values by the blue-shifted absorption feature of $\mathrm{H} \delta$.

The radial velocities inferred from the various regions in the red spectra are much more discrepant. Again, the velocities inferred from regions dominated by lines from the same multiplet are more similar to each other (for the two N II regions: $r m s$ of $9 \mathrm{~km} \mathrm{~s}^{-1} ; \chi_{\text {red }}^{2}=5.4$ ), while the velocities inferred from the $\mathrm{Al}$ III line are more different $\left(\mathrm{rms}\right.$ of $\left.9.7 \mathrm{~km} \mathrm{~s}^{-1} ; \chi_{\text {red }}^{2}=11\right)$.
In summary, we find that for the blue regions, the velocities inferred from the different lines track each other closely, but not exactly, while for the red region the correlation is much less tight. We stress, though, that no systematic differences are present. Indeed, below we will find that for all regions consistent radial-velocity amplitudes are inferred. Also, the differences from the best-fit radialvelocity curve will turn out to be substantially larger than the differences between the different lines, reflecting the fact that, to first order, the lines do behave similarly.

\section{The radial-velocity curve of HD 77581}

Ideally, the radial velocities reflect only the motion of the supergiant companion around the system's center of gravity, and are described by a function characterizing a Keplerian orbit with known period $P$, eccentricity $e$, periastron angle $\omega$, radial-velocity amplitude $K_{\text {opt }}$ and systemic velocity $\gamma$. We fitted the radial velocities derived for the different lines, leaving as free parameters only $K_{\text {opt }}$ and $\gamma^{1}$, since the other parameters are constrained very accurately by X-ray timing (Bildsten et al. 1997; see Table 4).

In practice, we are confronted with the fact that the individual radial-velocity measurements deviate significantly from the best-fit Keplerian orbit. This problem was already recognized in previous studies of Vela X-1. In Paper II, we found that these velocity "excursions" are correlated on a time scale of a day and must be nonorbital. This is the reason why we chose for the observational strategy to obtain one spectrum per night over many orbits of the system, with the expectation that the velocity excursions would average out, thus allowing an accurate determination of the radial-velocity amplitude.

In Fig. 12, we show the radial velocities derived from the two Si IV and the N III regions separately, as well as the radial-velocity curve derived from all these regions combined. Overlaid are the best-fit radial-velocity curves; the fitted radial-velocity amplitudes are listed in Table 3. One sees that there are large random velocity deviations from the Keplerian orbit, with root-mean-square deviations of $\sim 7 \mathrm{~km} \mathrm{~s}^{-1}$. Superposed on these random velocity excursions, there appear to be systematic deviations with orbital phase: the velocities are mainly above the best-fit curve between phases 0.15 and 0.25 and between 0.7 and 0.9 , and mainly below the fit between phases 0.45 and 0.65. We will return to this in Sect. 7.1.

The radial-velocity curve derived from the $\mathrm{H} \delta$ line (Fig. 13) shows much stronger systematic deviations with orbital phase, in particular an on average large negative velocity excursion between phases 0.45 and 0.65 (when the neutron star passes in front of the supergiant). As we discussed in Sect. 3.1, the $\mathrm{H} \delta$ profile is strongly distorted at these orbital phases, due to an additional blue

\footnotetext{
1 Since cross-correlation yields only relative velocities, the fitted value of $\gamma$ does not reflect the true systemic velocity of the binary.
} 

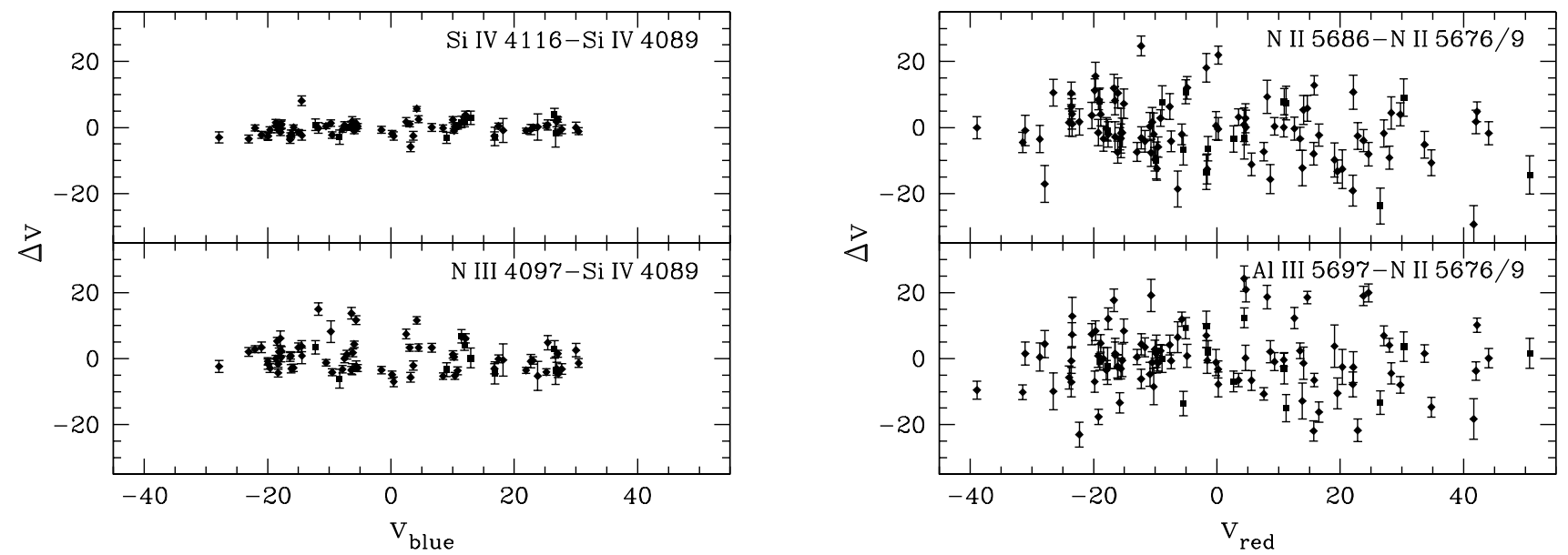

Fig. 11. Comparison of the radial velocities derived from different spectral lines. The difference in radial velocity is shown with respect to the radial velocity derived for one of the lines. The label at the top of each figure indicates which lines are involved. The left and right figures relate to the blue and red spectral domain, respectively.

absorption component. This component moves towards more negative velocities, also distorting the $\mathrm{N}$ III line profile. This likely is also the reason for the slight difference between the $\mathrm{N}$ III and Si IV radial-velocity curves. We did not include the region including $\mathrm{H} \delta$ in the final determination of the amplitude of the radial-velocity curve.

In Fig. 14, we show the radial-velocity curves derived from cross-correlation of the N II $5676,5679 \AA$, the N II $5686 \AA$, and the $\mathrm{Al}$ III regions, as well as of all regions combined. Fitted amplitudes are listed in Table 3. As mentioned, we did not use the $\mathrm{C}$ III $5710.77 \AA$ region because of possible contamination with the diffuse interstellar band.

From the figure, the scatter around the fitted radialvelocity curves is much larger than in the blue, with rootmean-square deviations of up to $20 \mathrm{~km} \mathrm{~s}^{-1}$ (Table 3 ). On top of this large scatter, systematic trends are harder to see, but in general the behaviour seems consistent with that in the blue.

We will return to the effects of the possible systematic effects with orbital phase below, after discussing the radial velocities inferred from ultraviolet spectra, about which there has been some controversy, and including these and other velocities in a combined velocity curve.

\section{Reanalysis of the IUE spectra}

A large number of ultraviolet spectra of HD 77581 have been obtained with the International Ultraviolet Explorer (IUE). In Paper II, we used these to determine radial velocities as well. We found relatively large uncertainties, of about $2.5 \mathrm{kms}^{-1}$, but given the large amplitude of the intrinsic variability, they nevertheless were a useful supplement to our optical observations.

After our study, a separate analysis of the IUE spectra was performed by Stickland et al. (1997). These authors included a larger set of spectra and determined velocities by cross-correlation with spectra of $\kappa$ Ori rather than with spectra of HD 77581 itself. They also used a somewhat different range of wavelength regions for calculating the cross-correlation profiles. The radial-velocity amplitude they derived is significantly smaller than that found by us; it is also inconsistent with that derived from optical spectra in Papers I and II. Clearly, in order to have confidence in our results, it is necessary to understand the origin of this discrepancy.

We have contacted Drs. Stickland and Lloyd and they kindly provided us with the IUE spectra (reduced using their procedures), as well as the procedures and wavelength regions used for normalization and crosscorrelation. We found that the main cause for the discrepancy was that in the Stickland et al. cross-correlation procedure for determining the stellar velocity, regions around interstellar and wind lines were zeroed in both programme-star and comparison-star spectra. Combined with slight inadequancies in normalization, this led to the introduction of artificial lines at fixed wavelengths in both spectra. In consequence, the radial velocities are biased systematically towards zero and the radial-velocity amplitude is underestimated. Dr. Stickland confirmed this conclusion, and reports that after correction of the procedure, the radial-velocity amplitude increases to $22 \mathrm{~km} \mathrm{~s}^{-1}$ (compared with the $17.8 \mathrm{~km} \mathrm{~s}^{-1}$ reported in their publication), thus solving the discrepancy.

In order to provide what we hope will be definitive radial velocities for the IUE spectra, we decided to re-do the complete analysis. For this purpose, we retrieved all 52 SWP spectra of HD 77581 from the archive. These archive spectra have been processed using NEWSIPS, the final IUE reduction pipeline. We determined radial-velocity differences between all pairs of images in the same way as done in Paper II, using the same wavelength regions (Table 4 in Paper II), and used these velocity differences to infer velocities for the individual spectra. Furthermore, we determined radial velocities relative to the spectra of $\kappa$ Ori used by Stickland et al. (1997): SWP 7745 (small aperture) and SWP 13776 (large aperture). 


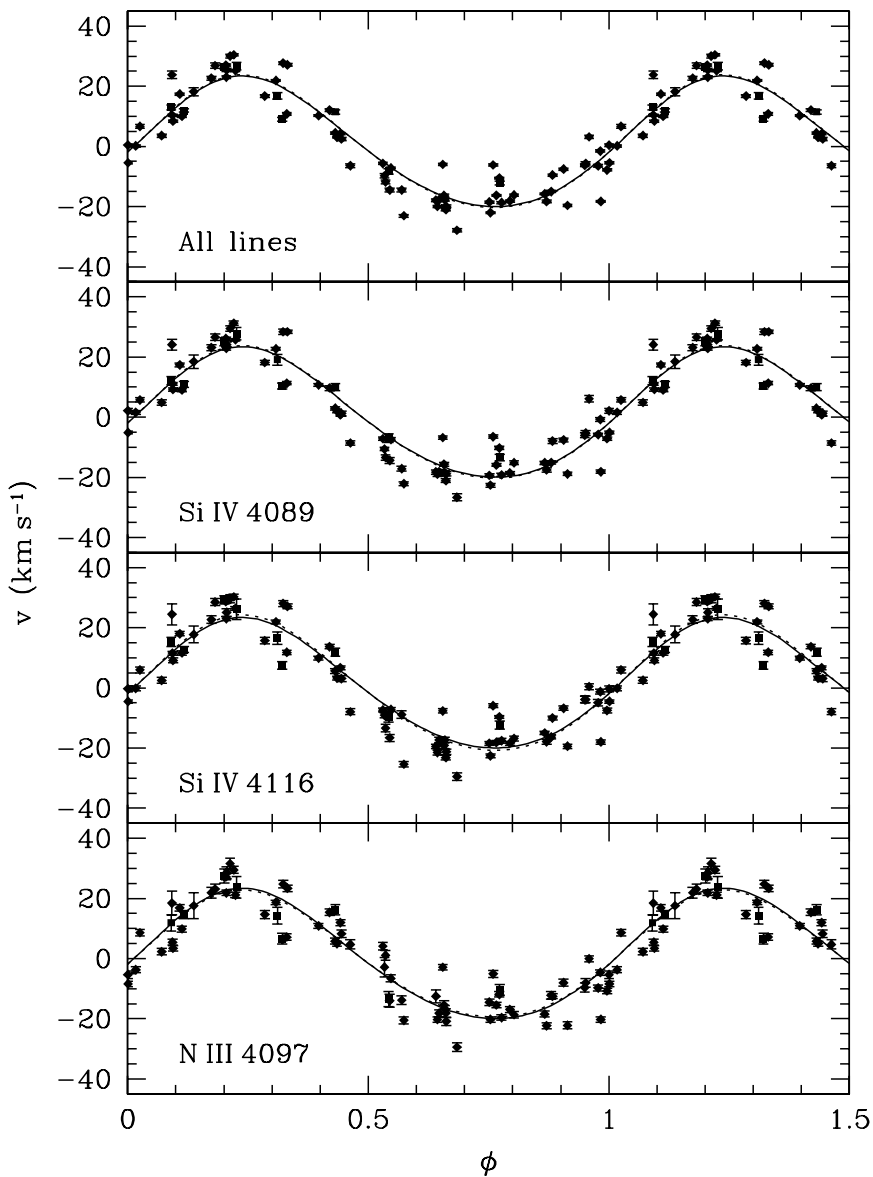

Fig. 12. Radial-velocity curves derived from the two Si IV and the N III regions separately, and one derived from the three regions combined (labelled "All lines"). The individual radialvelocity measurements (filled circles) with their formal error bars are shown together with the orbital fit to the curves (drawn line; free parameters for the fit are the relative $\gamma$ velocity and the radial-velocity amplitude $K$; see Table 3 ). The dotted line reflects the best-fit curve of Fig. 15.

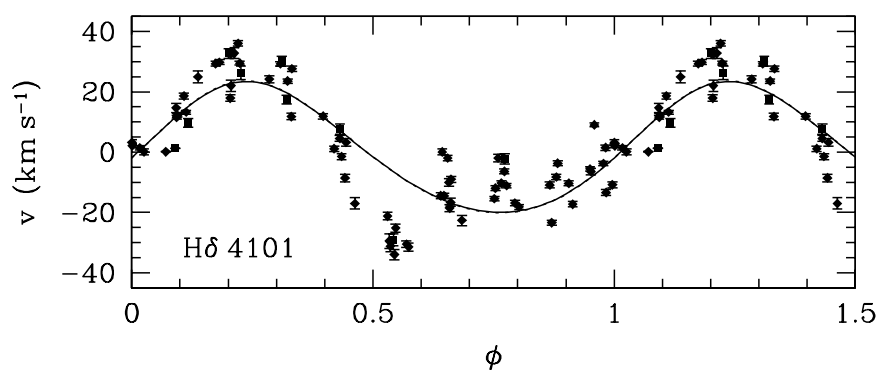

Fig. 13. Radial-velocity curve derived from the $\mathrm{H} \delta$ line. Note the large deviation towards negative velocities around orbital phase 0.5 due to the presence of a photo-ionisation wake.

We found that nine spectra of HD 77581 were of insufficient quality. For eight of these, the background was abnormally high (SWP 19041, 19062, 25762, 25763, 25847, $25848,25849,25851$ ), while for the remaining one, the integration was very short (1 ks; SWP 25850). We have not used any of these further.

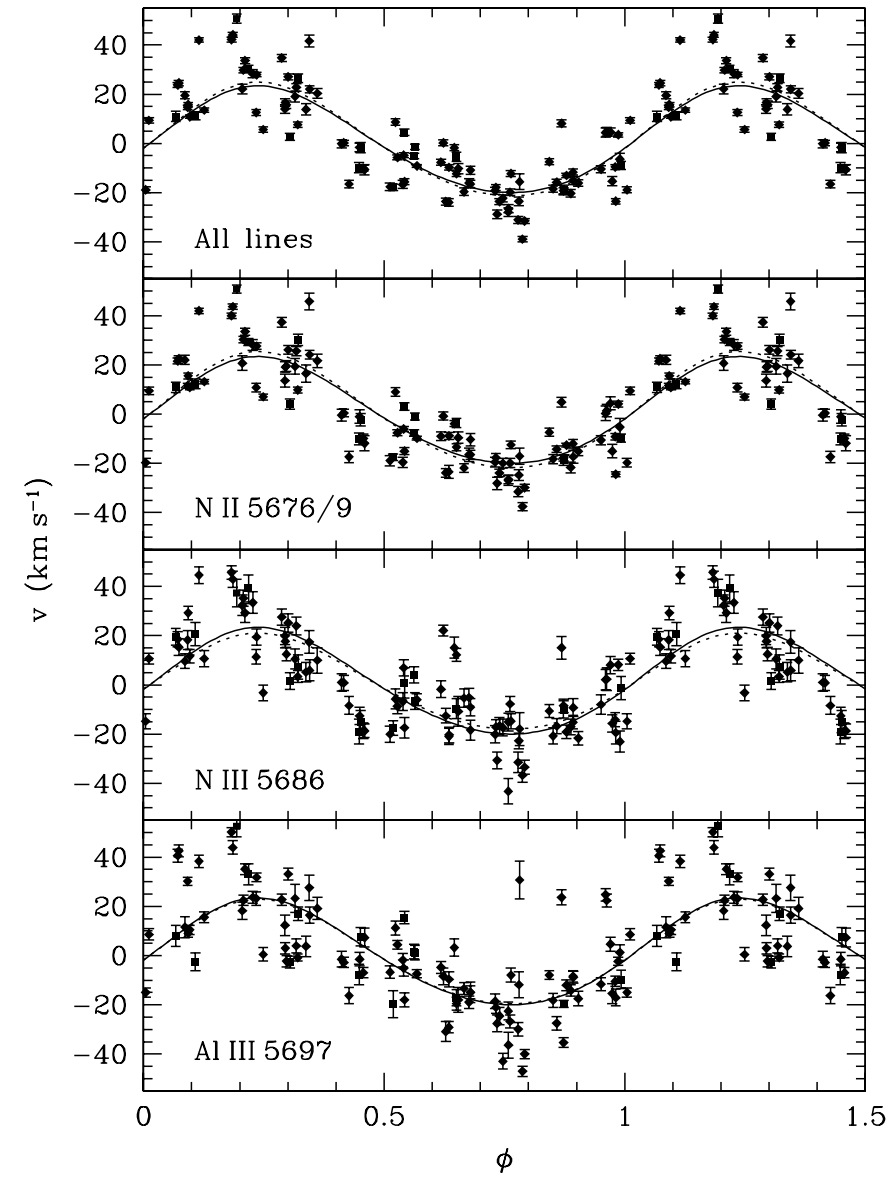

Fig. 14. Radial-velocity curves derived from each region separately and from all regions together for the red spectra.

In our analysis, it became clear that many of the crosscorrelation profiles show small excess peaks. These peaks, first found by Evans (1988) and discussed at some length in Paper II (see Fig. 4 in that paper) are thought to result from the presence of so-called fixed-pattern noise in the detector. Apparently, the new calibration employed in NEWSIPS has not resolved this. They occur only if the spectra are taken through the same aperture. As in Paper II, we corrected for such excess peaks by including an extra Gaussian peak in the fit to the discrete crosscorrelation profile (if spectra were taken through the same aperture).

In Table A.3 in the Appendix, we list the radial velocities derived from the cross-correlations of all spectra with each other. We verified that these are consistent with those derived from cross-correlation with $\kappa$ Ori. We prefer not to use the latter, however, because $\kappa$ Ori has a smaller $v \sin i$ than HD 77581, and thus is not a good template. We note that Stickland et al. (1997) argued that this actually makes it a better choice as a template. We do not agree: as one uses a template with narrower and narrower lines, approaching delta functions, the cross-correlation procedure reduces more and more to a weighted sampling of the line profile. Hence, the position of the peak of the cross-correlation profile will become a measurement 
Table 3. Radial-velocity amplitudes for the different datasets.

\begin{tabular}{|c|c|c|c|c|}
\hline Dataset & $N_{\mathrm{d}} / N_{\mathrm{n}}{ }^{\mathrm{a}}$ & $\mathrm{rms}^{\mathrm{b}}$ & Ampl. ${ }^{\mathrm{c}}$ & $K^{\mathrm{d}}$ \\
\hline Paper I & $82 / 29$ & $\ldots$ & $\ldots$ & $21.8 \pm 1.2$ \\
\hline Paper II, all data ${ }^{\mathrm{e}}$ & $79 / 50$ & 6.5 & 9.0 & $20.8 \pm 1.3$ \\
\hline CCD & $40 / 11$ & 4.5 & 11.3 & $21.9 \pm 3.1$ \\
\hline Photographic & $13 / 13$ & 4.0 & 6.8 & $21.5 \pm 1.9$ \\
\hline Blue, all lines & $77 / 75$ & 7.0 & 8.2 & $22.0 \pm 1.0$ \\
\hline Si IV $4089 \AA$ & & 7.1 & 8.3 & $22.0 \pm 1.0$ \\
\hline $\mathrm{N}$ III & & 7.4 & 8.8 & $21.1 \pm 1.1$ \\
\hline Si IV $4116 \AA$ & & 7.2 & 8.5 & $22.5 \pm 1.0$ \\
\hline Red, all lines & $104 / 97$ & 10.1 & 15.0 & $23.1 \pm 1.5$ \\
\hline N II $5676,5679 \AA$ & & 10.1 & 14.8 & $23.6 \pm 1.5$ \\
\hline N II $5686 \AA$ & & 11.3 & 18.2 & $19.5 \pm 1.9$ \\
\hline Al III $5697 \AA$ & & 11.8 & 19.9 & $21.2 \pm 2.0$ \\
\hline IUE & $43 / 40$ & 9.0 & 6.4 & $21.0 \pm 1.6$ \\
\hline CCD, Phot., Blue, IUE & $173 / 139$ & 8.4 & 8.3 & $21.7 \pm 0.8$ \\
\hline
\end{tabular}

a Total number of spectra and number of nights.

b Root-mean-square deviation (in $\mathrm{km} \mathrm{s}^{-1}$ ) from a Keplerian curve, including both measurement uncertainties and intrinsic deviations.

c Standard deviation (in $\mathrm{km} \mathrm{s}^{-1}$ ) of the normally distributed deviation amplitudes required to reproduce the rms and used to estimate the uncertainty in the radial-velocity amplitude (see text).

${ }^{\mathrm{d}}$ Inferred radial velocity amplitude (in $\mathrm{km} \mathrm{s}^{-1}$ ).

${ }^{\mathrm{e}}$ Including the velocities derived in Paper II for 26 IUE spectra. These are a subset of those analysed in Sect. 6 .

simply of the position of the deepest part of the line profile. In contrast, when one uses a template that matches in line width, all information in the line profile is used (in a way closely corresponding to $\chi^{2}$ minimalisation). We stress, however, that our result does not depend on this choice: from the radial velocities in Table A.3, we find $K_{\text {opt }}=21.0 \pm 1.6 \mathrm{~km} \mathrm{~s}^{-1}$ (Table 3 ), while from the velocities determined by cross-correlation with the small and large aperture spectra of $\kappa$ Ori, we derive $K_{\text {opt }}=22.5 \pm 2.0$ and $22.9 \pm 2.0 \mathrm{~km} \mathrm{~s}^{-1}$, respectively. Similarly, our results would change very little if we chose the wavelength regions used by Stickland et al. (1997) instead of the ones from Paper II.

\section{The combined radial-velocity curve and systematic effects with orbital phase}

To obtain the best measure of the radial-velocity amplitude, we combined the radial-velocity measurements of HD 77581 from our blue dataset with those derived from the IUE analysis and those obtained in Paper II from 13 photographic spectra and 40 CCD echelle spectra. We do not use the velocities derived from our red spectra, given their much larger scatter. The resulting radial-velocity curve is shown in Fig. 15. The best-fit radial-velocity amplitude is $K_{\text {opt }}=21.7 \pm 0.8 \mathrm{~km} \mathrm{~s}^{-1}$. In Table 3, we compare this with the values inferred in earlier sections from subsets of the data, as well as with those found previously. Clearly, the determinations are all consistent with each other.

\subsection{Search for systematic effects with orbital phase}

Our approach of obtaining radial velocities over an extended period of time was motivated by the hope that for a given orbital phase the large and highly significant radial-velocity deviations from a Keplerian orbit would average out. In other words, that just by adding measurements with random deviations, the accuracy of the derived radial-velocity amplitude, and thus the mass of the neutron star, would be improved. If there were to be systematic deviations with orbital phase, however, the measured mean radial velocity at a given orbital phase would not necessarily be equal to the Keplerian radial velocity of the star in its orbit.

We can test the above assumption by verifying whether the residuals binned over certain fractions of orbital phase are consistent with zero. In the lower panel of Fig. 15, we show the mean of the residuals determined for 9 phase bins, as well as the associated errors in the mean, calculated from the standard deviation in the phase bins. It is clear that these are not consistent with zero. The rootmean-square deviation of these phase-binned residuals is $2.7 \mathrm{~km} \mathrm{~s}^{-1}$, while only $1.35 \mathrm{~km} \mathrm{~s}^{-1}$ is expected based on the Monte-Carlo simulations. From those simulations, the likelihood that such a high value occurs by chance, under the assumption of no orbital-phase related systematic effects, is smaller than $0.1 \%$.

Another piece of evidence that systematic, phaselocked effects occur, is that the eccentricity one infers from the optical data is 0.18 (and $\omega=355^{\circ}$ ). From our simulations, the probability to find a value this much larger than the BATSE value is less than $1 \%$.

Understanding the physical origin of these systematic deviations might allow one to account for them and to further improve the accuracy of the determination of the radial-velocity amplitude. We discuss three possible mechanisms below.

\subsection{Wind effects and evidence for the existence of a photoionisation wake}

In the orbital phase range $0.45-0.65$, strong velocity excursions are observed in the radial-velocity curve obtained for the $\mathrm{H} \delta$ line (Sect. 4). In this phase interval, the X-ray source passes through the line of sight of the B supergiant. Inspection of the $\mathrm{H} \delta$ line shows that at these orbital phases the absorption profile includes an additional blueshifted absorption component, similar to what is observed in time series of spectral lines formed in the supergiant's stellar wind (e.g., Kaper et al. 1994). In consequence, the cross-correlation procedure yields a more negative radial velocity for the $\mathrm{H} \delta$ line. 


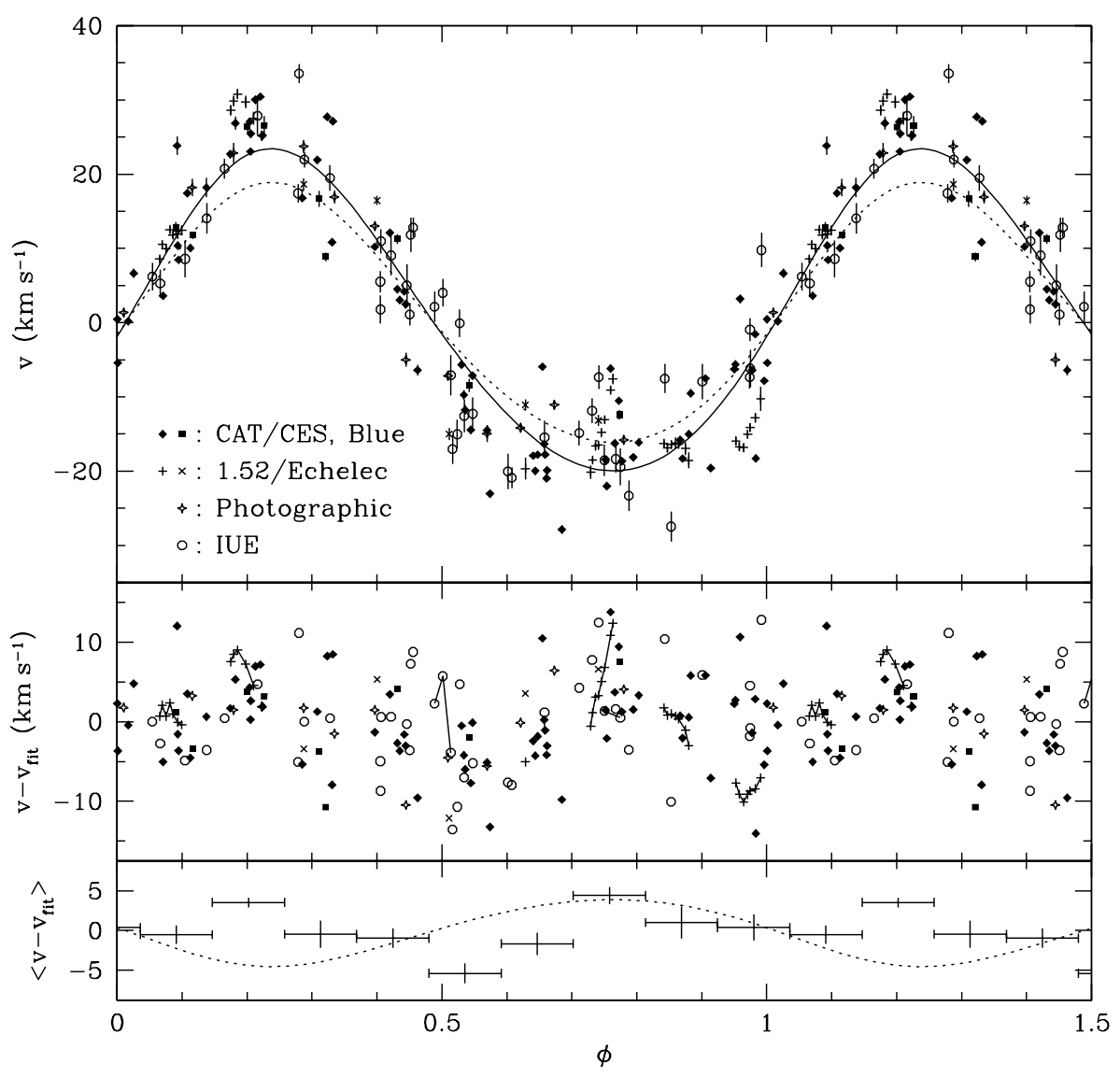

Fig. 15. Radial-velocity curve derived from the combined data of this paper and Paper II (top panel). Overdrawn is the Keplerian curve that best fits the nightly averages of the data (solid line), as well as the curve expected if the neutron star has a mass of $1.4 M_{\odot}\left(\right.$ dotted line; $\left.K_{\mathrm{opt}}=17.5 \mathrm{~km} \mathrm{~s}^{-1}\right)$. The residuals to the best-fit are shown in the middle panel. For clarity, the error bars have been omitted. Points taken within one night are connected with lines. In the bottom panel, the residuals averaged in 9 phase bins are shown. The horizontal error bars indicate the size of the phase bins, and the vertical ones the error in the mean. The dotted line indicates the residuals expected for a $1.4 M_{\odot}$ neutron star.

The origin of this additional blue-shifted absorption component is likely a so-called photo-ionization wake. The X-ray photons emitted by the compact X-ray source fully ionize the surrounding wind regions, creating an extended Strömgren zone in the stellar wind. The presence of such a zone was predicted by Hatchett \& McCray (1977), and can explain the observed strong orbital modulation of the ultraviolet resonance lines of HD 77581 (e.g., Kaper et al. 1993). Due to the high level of ionization of the plasma contained in the Strömgren zone, inside this zone the radiative acceleration of the stellar wind is quenched, leading to low wind velocities within the Strömgren zone. Therefore, at the trailing border of the Strömgren zone a strong shock is formed where the relatively fast ambient flow meets the slow wind that moved through the Strömgren zone. The formation of such a "photo-ionization wake" is clearly seen in hydrodynamical simulations (Blondin et al. 1990). Additional evidence for the existence of such a structure was found in the X-ray light curve of Vela X-1 (Feldmeier et al. 1996).

On two occassions, in February and May 1996, we obtained spectra of strong optical lines formed in the stellar wind together with some of the (red) spectra that have been used to measure the star's radial velocity. In the period 10-18 February 1996 we monitored the $\mathrm{H} \alpha$ line of HD 77581; Fig. 16 shows the dramatic (wind) variability observed in this line. At phase 0.54 a strong, blue-shifted $\left(-200\right.$ to $\left.-300 \mathrm{~km} \mathrm{~s}^{-1}\right)$ absorption component appears, which at later phases migrates towards more negative velocities. Another blue-shifted absorption component, most probably the remnant of a previous passage of the X-ray source, is seen in the first spectra obtained $(\phi=0.09$, $0.20,0.32)$. The emission component of the $\mathrm{H} \alpha$ profile is variable as well; notice the blue-shifted emission component present at phase $0.3-0.4$, most likely caused by emission from the dense photo-ionization wake when the X-ray source is approaching the line of sight. For comparison, Fig. 16 also displays the red spectra obtained within the same nights, where zero velocity corresponds to the rest wavelength (in the heliocentric frame) of the $\mathrm{N}$ II line at $5679.56 \AA$. This line profile clearly demonstrates intrinsic variability which cannot be solely due to orbital motion. However, apart from the enhanced blue-shifted absorption at $\phi=0.54$, there does not seem to be an obvious 

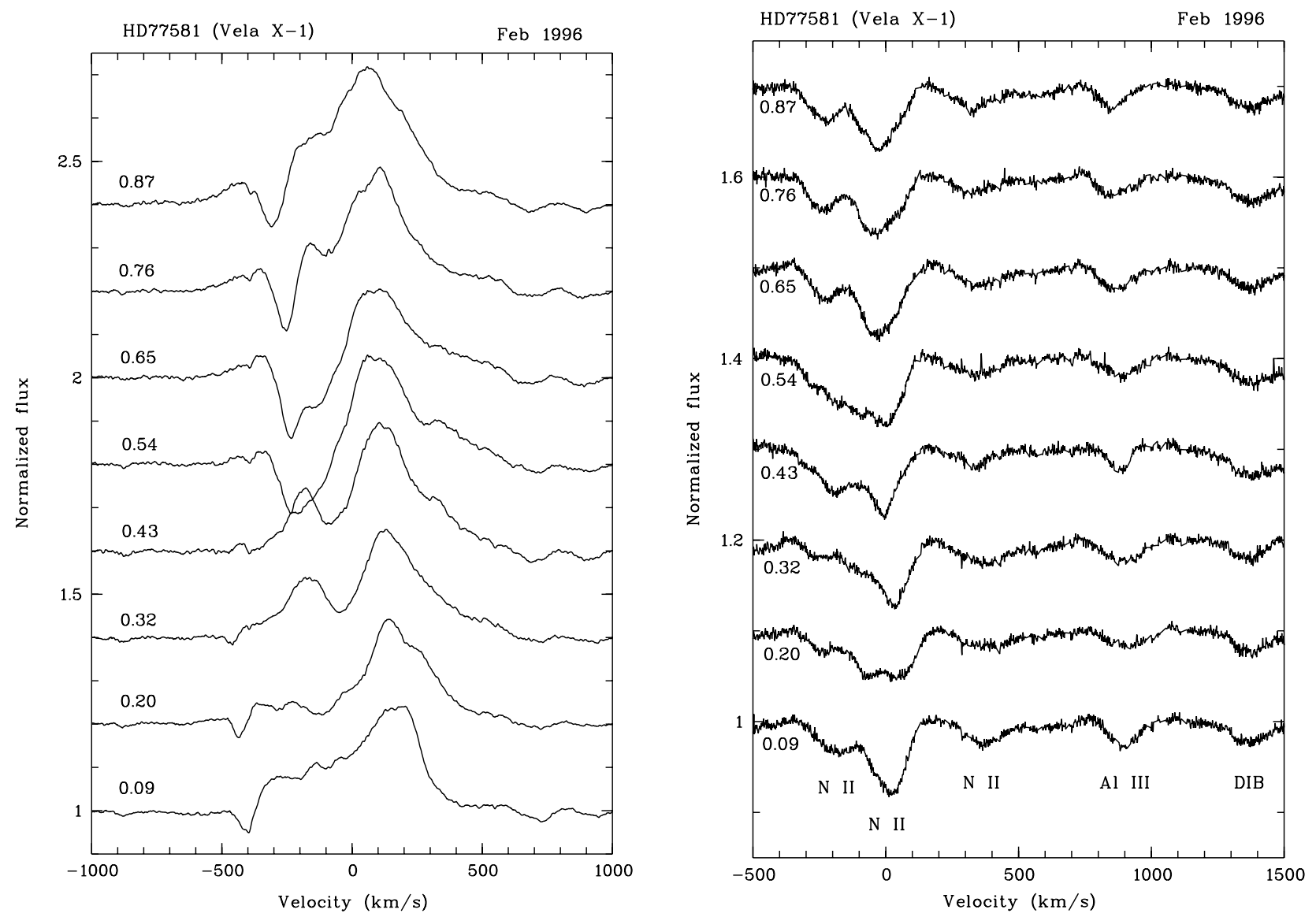

Fig. 16. Sequence of $\mathrm{H} \alpha$ profiles (left) obtained in February 1996, together with the simultaneously taken red spectra.

correlation between the variability in the $\mathrm{H} \alpha$ and the $\mathrm{N}$ II lines. Remarkable is the shallow (incipient emission?) and broad (additional blue-shifted absorption component?) absorption profile observed at phase 0.2 .

From May 10 to 16 , we monitored the He I $5876 \AA$ line, which is not as strong as the $\mathrm{H} \alpha$ line, but is still sensitive to changes in the base of the stellar wind. The systematic changes (Fig. 17) strongly resemble those observed in the $\mathrm{H} \beta$ line (Kaper et al. 1994) and are similar to the variations found in $\mathrm{H} \alpha$. Comparison with the red N II spectra shows that at $\phi=0.34$ both the He I and the N II (and $\mathrm{Al}$ III) lines are shallower, while at $\phi=0.57$ all lines are deeper. The blue-shifted absorption is enhanced at phases 0.46 and 0.56 .

We conclude that the systematic deviation in radial velocity of the $\mathrm{H} \delta$ line in the phase interval $0.45-0.65$ is caused by the presence of a photo-ionization wake. Weaker spectral lines are much less strongly, though still measurably affected in this phase interval; stronger lines like $\mathrm{H} \alpha$ show large deviations in line shape over a much wider range of the orbit. We decided to leave out the radialvelocity measurements based on the $\mathrm{H} \delta$ line in our further analysis. In Sect. 7.5, we investigate the impact of the points between phase 0.45 and 0.65 on the determination of the radial-velocity amplitude. It turns out to be small.

\subsection{Tidal distortion}

Tidal deformation could also cause systematic deviations of the radial-velocity curve, since for a deformed star "center-of-light" radial-velocity measurements do not necessarily reflect the actual center-of-mass velocity. To investigate the effect this might have on inferred orbital parameters, Van Paradijs et al. (1977b) made model calculations for a star with properties appropriate for HD 77581. For simplicity, they assumed a circular orbit and synchronous rotation of the primary, so that the shape of the star was given by a single Roche equipotential surface at all phases. Using theoretically predicted line profiles, including the Si IV 4089 line, they find that appreciable systematic effects can occur, differing from ion to ion, with the main expected deviations being a positive one around orbital phase 0.1 and a negative one around phase 0.9 . As a result, an apparent eccentricity may arise and the radial-velocity amplitude may be overestimated, in unfavourable cases by up to $30 \%$.

The systematic deviations we encounter in the radialvelocity curve behave differently. The larger deviations occur around orbital phases 0.2 and 0.8 , and at both phases the deviations are directed towards positive velocities. Thus, it seems the effects of the tidal deformation are not as large as predicted. One should keep in mind, however that the assumptions made by 

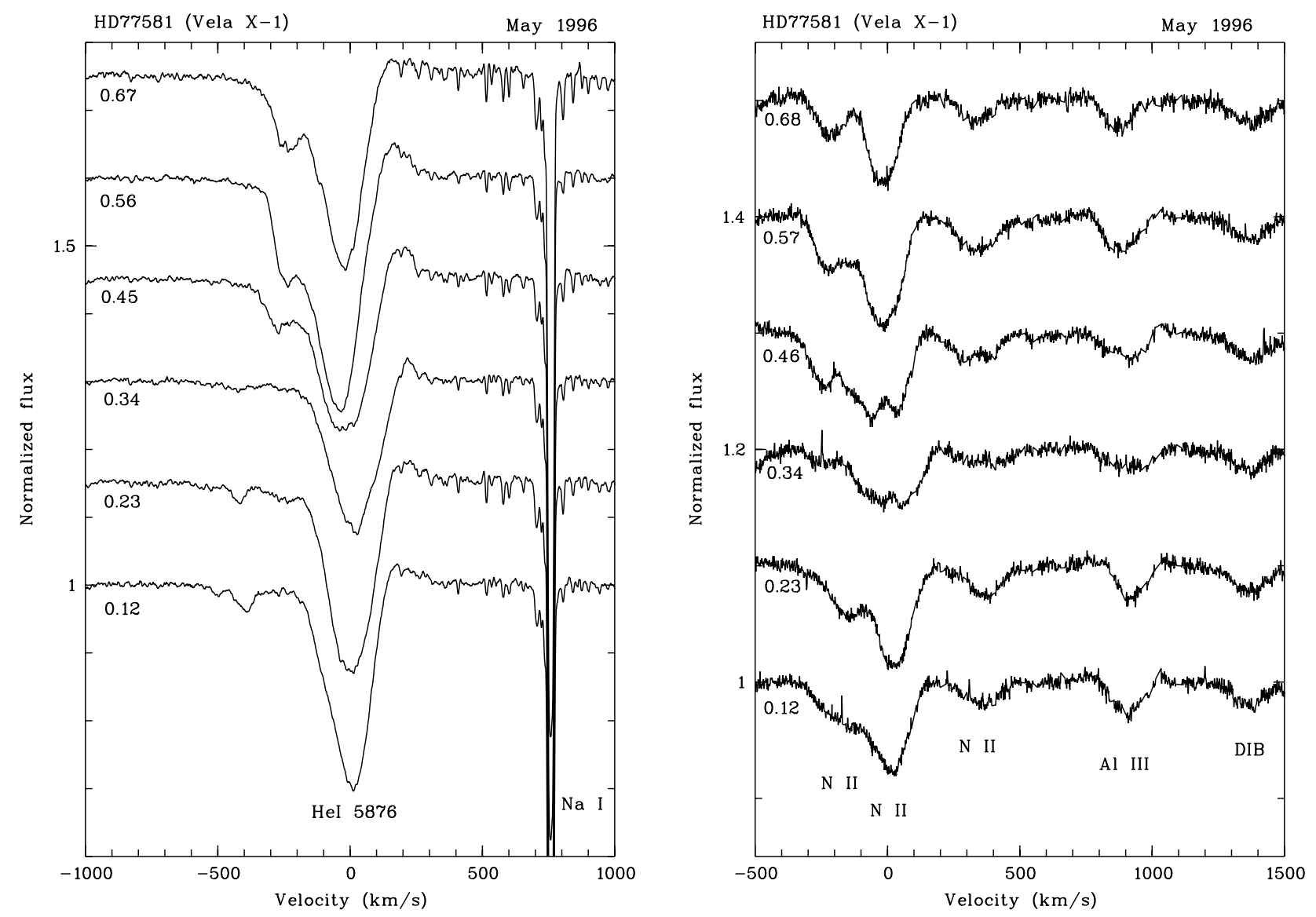

Fig. 17. Sequence of He I profiles (left) obtained in May 1996 compared to the simultaneously taken red spectra.

Van Paradijs et al. (1977b) do not correspond to the actual situation in Vela X-1: the orbit is eccentric and HD 77581 rotates sub-synchronously (by a factor of about $2 / 3$, Zuiderwijk 1995). Given the possibly large effects on the inferred parameters, it would be worthwile to reconsider these issues.

\subsection{Non-radial pulsations}

Apart from distorting the shape of the star, tidal forces can excite (non-radial) pulsations. With a neutron star in a close eccentric orbit (the distance between the neutron star and the non-synchronously rotating surface of HD 77581 is about half a stellar radius) one can expect that tidal waves are excited at the surface of HD 77581 (e.g., Witte \& Savonije 1999). The neutron star's orbital frequency may resonate with certain non-radial modes, perhaps phase-locked with the orbit. The properties of these modes depend on the details of the internal structure of the supergiant, which has undergone a complex history of binary evolution, including a phase of mass transfer and a nearby supernova explosion forming the neutronstar companion.

Radial and low-degree non-radial pulsations can change the shape of photospheric absorption line profiles significantly (e.g., Vogt \& Penrod 1983). This is due to local temperature variations induced by the pulsations and/or local Doppler shifts related to the 3-dimensional pulsation velocity field.

The question is whether such tidally induced (or selfexcited) pulsations, when present in HD 77581, are detectable through inspection of the detailed shape of the line profiles and, if so, could result in systematic deviations in the radial-velocity curve. According to Telting \& Schrijvers (1997), low-order pulsation modes (with degree $\ell<3$ ) can lead to detectable radial-velocity variations and changes in the shape of the line-profiles. For instance, moment analyses of the spectral line profiles (Balona 1986; Aerts et al. 1992) have been successfully used to detect low-order modes in single pulsators such as the $\beta$ Cephei stars.

If the presence of non-radial pulsations is the physical cause for the observed deviations in radial velocity, knowledge of the pulsation mode(s) might provide a tool to "correct" the measured radial velocities and to improve the accuracy of the determination of the Keplerian orbit of HD 77581. However, a mode can only be identified if the time sampling of the data is sufficient to resolve its periodicity (in practice several spectra per hour and continuous - 24 hours per day - coverage). The time sampling of the spectra used in this paper (one spectrum per day) is poor; it is sufficient to cover the change in radial velocity due 
to orbital motion, but likely insufficient to identify pulsation modes. Another disadvantage of the current dataset is the limited spectral coverage; this excludes the possibility to combine many different spectral lines in order to increase the signal-to-noise ratio and to separate line changes induced by the temperature- and velocity distribution associated with a given pulsation.

A preliminary moment analysis performed on the line profiles comprised in our spectra did not yield a useful result. This is an indication that any putative non-radial pulsation modes are not phase-locked to the orbital motion and/or do not persist for a long period of time. A dataset obtained with better time sampling and spectral coverage is required to further investigate the occurrence of non-radial pulsations in the atmosphere of HD 77581 .

\subsection{Orbital-phase intervals excluded}

In the $\mathrm{H} \delta$ profile, we found clear evidence for an ionisation wake in the phase interval between 0.45 and 0.65 . It may be that the photoionisation wake has a (less obvious) effect on the other spectral lines as well. We can verify to what extent the exclusion of this phase interval would affect the amplitude of the radial-velocity curve (and thereby the measurement of the neutron-star mass). Similarly, in previous studies it was found that the largest deviations occurred near velocity minimum (Paper II and references therein). From Fig. 15, one sees that the phase bin at velocity minimum again has one of the largest and most significant deviations from zero.

In order to see what is the effect of these phase intervals on the solution, we decided to exclude, like in Paper II, all possible one ninth and two ninth cycle wide orbitalphase intervals (i.e., approximately one and two-day wide) from the fit to the radial-velocity curves, starting from orbital phase zero until one with a step of one ninth. Subsequently, we fitted the remaining points and derived the amplitude of the radial-velocity curve, as well as the uncertainty estimated using our Monte-Carlo simulations. The resulting amplitude is shown as a function of the excluded phase intervals in Fig. 18. Also shown is the rms of the phase-binned residuals. If a specific phase interval were responsible for virtually all of the phase-locked deviation, one would expect that the binned residuals would become consistent with zero when that phase bin is excluded. From the figure, one sees that excluding the bins near inferior conjunction of the neutron star, when the ionisation wake is most important, and near velocity minimum have the largest effect on the rms. Excluding inferior conjunction, the inferred radial-velocity amplitude is not affected much, since the velocities are at a zero crossing, while excluding velocity minimum, a much larger velocity amplitude is inferred; indeed, excluding this bin leads to the only change in radial-velocity amplitude whose probability of occurring by chance, as inferred from the simulations, is less than $1 \%$.
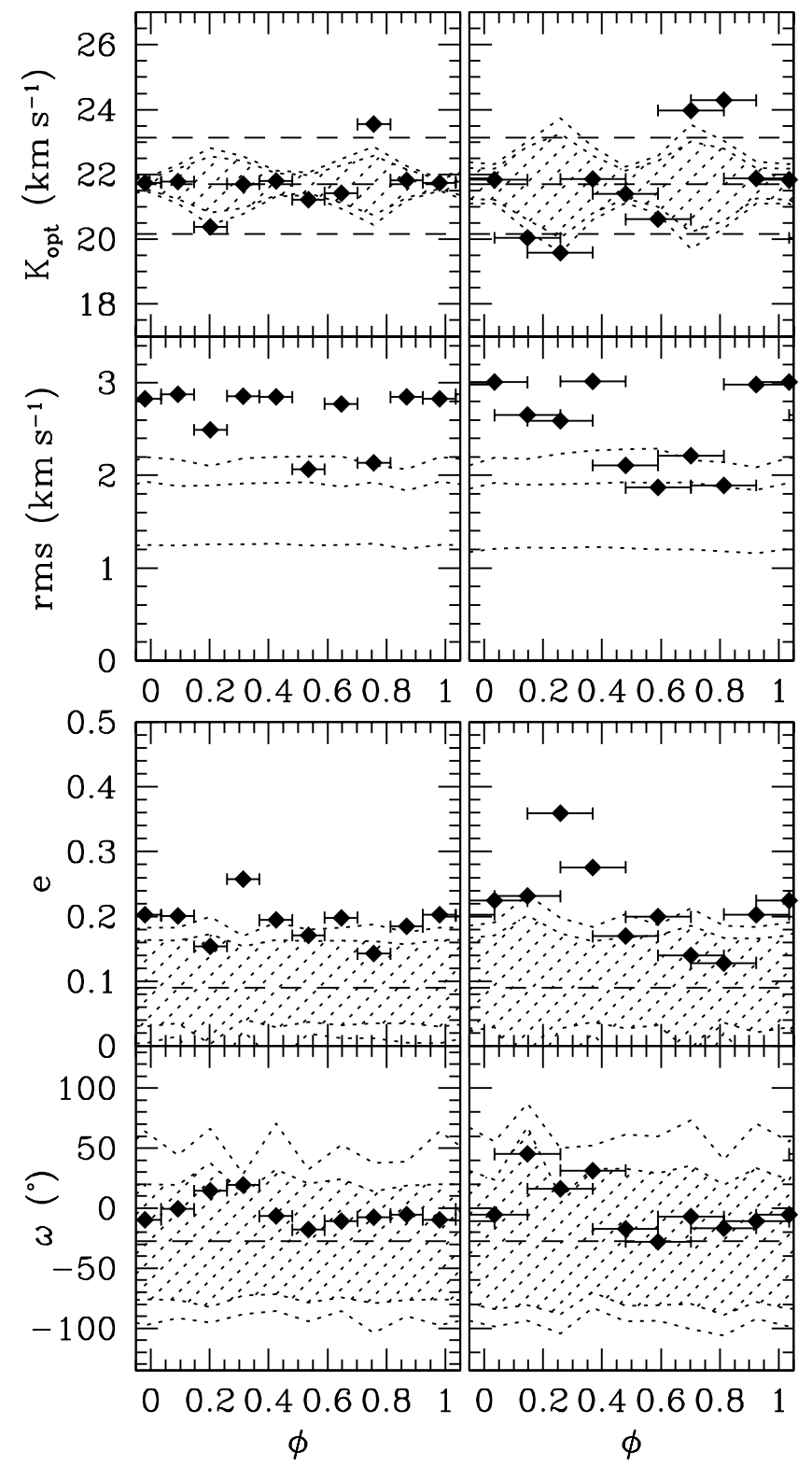

Fig. 18. Results for fits to the radial-velocity curve with phase intervals of width $1 / 9$ (left) and 2/9 (right) excluded. The points in the top panel show the best-fit radial-velocity amplitudes found when the phase range indicated by the horizontal error bars is excluded. The short-dashed contours indicate the $95 \%$ and $99 \%$ confidence range of the variation expected from our simulations (for which it is assumed that the variability of the star is independent of orbital phase.) The long-dashed lines indicate the best-fit amplitude to all data and the associated 95\% confidence uncertainties. The panels in the second row show the root-mean-square deviation of the phase-binned residuals. The short-dashed lines indicate the levels below which $50 \%, 95 \%$, and $99 \%$ of the simulated data are contained. In the bottom two rows of panels, the best-fit values for the eccentricity and periastron angle are shown. The long-dashed lines indicate the accurate values inferred from the BATSE measurements (Table 4), the short-dashed contours the $95 \%$ and $99 \%$ ranges expected from the simulations. 


\subsection{Fitting eccentricity and periastron angle}

In another attempt to determine whether any specific bin is responsible for the phase-locked systematic effects, we repeated the above simulations but with this time the eccentricity and the periastron angle as free parameters in the fitting procedure. In principle, of course, one should reproduce the values found from the $\mathrm{X}$-ray analysis. The bottom panels of Fig. 18 show the resulting eccentricity and periastron angle. We find that when early phase intervals are excluded, the inferred eccentricity remains far larger than the true value, measured by Bildsten et al. (1997; Table 4). When phase intervals around velocity minimum are excluded, however, the inferred eccentricity becomes much closer to the true value.

\subsection{The uncertainty in the radial-velocity amplitude}

The above results suggest that the main systematic effects on the radial-velocity curve occur near inferior conjunction and at velocity minimum. If so, the radial-velocity amplitude inferred from all the data likely is an underestimate of the true value. However, while we believe the velocity deviation at inferior conjunction can be understood - in terms of a photo-ionisation wake - we do not have a good idea for the cause of the other systematic deviations. Lacking this, it is difficult to estimate the uncertainty that is introduced. Probably best is to assume there is no bias to high or low radial-velocity amplitude, and base the estimate on the increase in the uncertainties required to match the observed root-mean-square deviations of the phase-binned residuals. This is about a factor two, and corresponds to a similar increase in the uncertainty on the final radialvelocity amplitude. In consequence, our final estimate is not much more precise than values given previously, in which the influence of systematic effects with orbital phase was not taken into account. We conclude that our best present estimate is $K_{\mathrm{opt}}=21.7 \pm 1.6 \mathrm{~km} \mathrm{~s}^{-1}$.

\section{The mass of Vela $X-1$}

From the ratio of the radial-velocity amplitudes of the optical and X-ray components, we infer a mass ratio

$q \equiv M_{\mathrm{X}} / M_{\mathrm{opt}}=K_{\mathrm{opt}} / K_{\mathrm{X}}=0.078 \pm 0.006$,

after inserting the value of the radial-velocity amplitude of the X-ray source (derived from X-ray pulse-timing analysis)

$K_{\mathrm{X}} \equiv(2 \pi / P) a_{\mathrm{X}} \sin i\left(1-e^{2}\right)^{-1 / 2}=278.1 \pm 0.3 \mathrm{~km} \mathrm{~s}^{-1}$.

Combined with the X-ray mass function

$f_{\mathrm{X}} \equiv \frac{M_{\mathrm{X}}^{3} \sin ^{3} i}{(1+1 / q)^{2}}=\frac{4 \pi^{2}}{G P^{2}}\left(a_{\mathrm{X}} \sin i\right)^{3}=19.74 \pm 0.07 M_{\odot}$, we infer

$M_{\mathrm{X}} \sin ^{3} i=1.78 \pm 0.15 M_{\odot}$, and

$M_{\mathrm{opt}} \sin ^{3} i=22.9 \pm 0.3 M_{\odot}$.

The above numbers are lower limits to the actual masses, since the inclination can only be smaller than $90^{\circ}$. As in Paper II, we use the method introduced by Joss \& Rappaport (1983) to estimate the inclination $i$, the semimajor axis $a$, the radius $R_{\text {opt }}$ and mass $M_{\text {opt }}$ of the companion, the co-rotation factor $f_{\text {co }}$, and the neutron-star mass $M_{\mathrm{X}}$, from the observed values of the orbital period $P$, eccentricity $e$, periastron angle $\omega$, projected semimajor axis of the neutron star $a_{\mathrm{X}} \sin i$, eclipse duration $\theta_{\text {ecl }}$, radial-velocity amplitude $K_{\mathrm{opt}}$, and projected rotational velocity $v_{\text {rot }} \sin i$ (see Table 4 ). The corresponding uncertainties are found by means of a Monte-Carlo error propagation technique, in which a large number of trial evaluations are made for parameters drawn from the observed uncertainty distribution (which are assumed to be normal, except for $\theta_{\text {ecl }}$, for which a uniform distribution is used). Following Joss \& Rappaport (1983), we assume that the star is close to filling its Roche lobe, with a volume filling factor between 90 and $100 \%$ at periastron.

The results are listed in Table 4; for the mass of the neutron star, we find

$M_{\mathrm{X}}=1.86 \pm 0.16 M_{\odot}$.

Here we should add a note of caution: as in Paper II, for a substantial fraction $(37 \%)$ of the trials, the randomlychosen parameters are inconsistent with each other in the sense that the eclipse duration cannot be reproduced for any inclination. We discard these trials, but note that as a result, the inferred parameters are based on effective distributions of the input parameters which no longer reflect the actual ones. Most likely, this is because the assumption that the star is in instantaneous hydrostatic equilibrium is incorrect (see Paper II for a more extensive discussion).

Given the above uncertainty, and given that for constraints on the neutron-star equation of state, the most interesting result is a firm lower limit to the mass of the neutron star, we prefer to give lower limits in terms of $M_{\mathrm{X}} \sin ^{3} i$. For these, we use the one-sided $95 \%$ and $99 \%$ confidence lower limits to the radial-velocity amplitude, of 19.1 and $17.9 \mathrm{~km} \mathrm{~s}^{-1}$, respectively, for which we infer $M_{\mathrm{X}} \sin ^{3} i>1.54$ and $1.43 M_{\odot}$, respectively.

\section{Ramifications}

The best estimate of the mass of Vela X-1 is $1.86 M_{\odot}$. Unfortunately, no firm constraints on the equation of state are possible, since systematic deviations in the radialvelocity curve do not allow us to exclude a mass around $1.4 M_{\odot}$ as found for other neutron stars. Therefore, we will not discuss the equation of state here, but only address evolutionary ramifications of what still most likely is a massive neutron star, assuming that the equation of 
Table 4. Parameters of the Vela X-1/HD 77581 system.

\begin{tabular}{|c|c|c|c|}
\hline Parameter & Value & Unit & Ref. \\
\hline \multicolumn{4}{|c|}{ Observed ( $1 \sigma$ uncertainties) } \\
\hline$T_{\pi / 2}^{\mathrm{a}}$ & $2448895.7186(12)$ & JD & Bildsten et al. (1997) \\
\hline$P_{\text {orb }}$ & $8.964368(40)$ & day & \\
\hline$a_{\mathrm{X}} \sin i$ & $113.89(13)$ & lt-s & \\
\hline$e$ & $0.0898(12)$ & & \\
\hline$\omega_{\mathrm{X}}$ & $152.59(92)$ & $\circ$ & \\
\hline$\theta_{\mathrm{ecl}}^{\mathrm{b}}$ & $30-36$ & $\circ$ & See Paper I \\
\hline$v_{\text {rot }} \sin i$ & $116(6)$ & $\mathrm{km} \mathrm{s}^{-1}$ & Zuiderwijk (1995) \\
\hline$K_{\mathrm{opt}}$ & $21.7(16)$ & $\mathrm{km} \mathrm{s}^{-1}$ & This paper \\
\hline \multicolumn{4}{|c|}{ Derived ( $\sigma$ uncertainties) } \\
\hline$M_{\mathrm{X}} \sin ^{3} i$ & $1.78 \pm 0.15$ & $M_{\odot}$ & \\
\hline$M_{\mathrm{opt}} \sin ^{3} i$ & $22.9 \pm 0.3$ & $M_{\odot}$ & \\
\hline \multicolumn{4}{|c|}{ Inferred (95\% confidence ranges ${ }^{\mathrm{c}}$ ) } \\
\hline$f_{\mathrm{co}}$ & $0.69_{-0.08}^{+0.08}$ & & \\
\hline$i$ & $>73$ & $\circ$ & \\
\hline$a$ & $53.6_{-0.9}^{+1.7}$ & $R_{\odot}$ & \\
\hline$R_{\mathrm{opt}}$ & $30.4_{-2.1}^{+1.6}$ & $R_{\odot}$ & \\
\hline$M_{\mathrm{opt}}$ & $23.8_{-1.0}^{+2.4}$ & $M_{\odot}$ & \\
\hline$M_{\mathrm{X}}$ & $1.86_{-0.32}^{+0.32}$ & $M_{\odot}$ & \\
\hline
\end{tabular}

a Time of mean longitude $90^{\circ}$.

b $99 \%$ confidence range.

${ }^{\text {c }}$ For the inferred quanties, 95\% confidence ranges are listed, since the uncertainties on many parameters have highly nonnormal distributions. The one exception is the mass of the neutron star, whose uncertainty distribution is close to normal. Hence, the associated $1 \sigma$ error is about $0.16 M_{\odot}$.

state is sufficiently stiff for such a massive neutron star to exist.

One reason that the high mass is peculiar (if true), is that the most accurately determined neutron-star masses fall in a surprisingly small range: for the binary radio pulsars with neutron-star companions, the masses are in the range $1.338-1.442 M_{\odot}$. Indeed, Thorsett \& Chakrabarty (1999) found that all binary radio pulsars have masses which are consistent with a normal distribution around $1.35 M_{\odot}$, with the remarkably small spread of $0.04 M_{\odot}$. Furthermore, for all X-ray binaries other than Vela X-1 for which dynamical masses are available, the neutron-star masses are consistent with the same value (Van Kerkwijk et al. 1995a).

Obviously, since for no equation of state masses substantially lower than $1.4 M_{\odot}$ are excluded, at least the lower limit to the above narrow range must be set by the way in which the neutron star is formed, i.e., by the physics of supernova explosions and the evolution of stars massive enough to reach core collapse. This leads one to wonder whether it could not be the formation mechanism on its own that leads to the narrow range in mass, just as for white dwarfs the formation leads to masses mostly within a very narrow range around $0.6 M_{\odot}$, well below the Chandrasekhar mass.

If the formation indeed leads to a narrow range of masses, high masses could arise only if substantial amounts of matter are accreted. This is not expected to have occurred for any of the radio or X-ray pulsars with accurate mass estimates. For low-mass X-ray binaries, in which substantial accretion is expected to have occurred, masses around $2 M_{\odot}$ have been suggested (see, e.g., Orosz \& Kuulkers (1999) for an analysis of Cyg X-2, and Zhang et al. (1997) for inferences based on quasi-periodic oscillations). All these estimates, however, rely to greater or lesser extent on unproven assumptions. Furthermore, for the putative descendants, radio pulsars with white dwarf companions, there is no evidence for such high masses (Thorsett \& Chakrabarty 1999 and references therein).

From recent evolutionary calculations, Timmes et al. (1996) indeed expect that massive stars in close binaries, which explode as Type Ib supernovae, give rise to initial neutron star masses in a narrow range around $1.3 M_{\odot}$. This value does not include subsequent mass accretion from a reverse shock or from a massive component in a binary system, and Timmes et al. expect that the final masses could be somewhat higher. Interestingly, for single stars, which explode as Type II supernovae, they found a bimodal distribution of initial neutron-star masses, with narrow peaks at 1.27 and $1.76 M_{\odot}$. As mentioned, they did not find a bimodal distribution for stars in close binaries, but at present it is not clear whether this result will hold (Woosley 2000, private communication). If stars in close binaries turn out to be more similar to single stars after all, one could assign most neutron stars to the first peak, and Vela X-1 to the second.

We end by noting that even if neutron stars are formed with masses in two peaks, it might be rather unlikely to find a massive neutron star in a close double neutron star binary. This is because such systems require a commonenvelope stage, in which a merger can only be avoided if the initial orbit was very wide. Stars massive enough to form a massive neutron star, however, likely do not evolve through a red-giant phase, and a common-envelope phase would occur only for rather close orbits, for which the binary would merge.

Acknowledgements. The authors wish to thank the ESO La Silla Observatory staff for their dedicated efforts in executing this difficult programme. Without the help of the night assistants at the CAT (and sometimes of the observers themselves whose programme got interrupted) this programme would not have been possible. A special word of thanks goes to Jesus Rodriguez, the night operator at ESO Headquarters who carried out the remote part of the observations from Garching. We thank the referee, Dr. Phil Charles, for carefully reading the manuscript and for his suggestions that helped to improve the paper. OB acknowledges the support of an ESO studentship. L. K. and M. Hv. K. are supported by fellowships of the Royal Netherlands Academy of Arts and Sciences. 
Table A.1. Observing log and radial velocities for the blue spectra (central wavelength $4102 \AA$ ). The second digit of the identification (first column) specifies the CCD used (b4 for CCD\#34 and b8 for CCD\#38). In the second column the barycentriccorrected Julian Date of each observation is listed, and in the third the orbital phase calculated using the ephemeris of Bildsten et al. (1997; see Table 4). The following four columns list radial velocity and associated uncertainty (in $\mathrm{km} \mathrm{s}^{-1}$ ) for each spectral region separately, as derived by means of cross-correlation. The last columns lists radial velocity and uncertainty derived from the cross-correlation of the ensemble of the two Si IV and the N III regions.

\begin{tabular}{cccccccc}
\hline Ident & BJD $_{\text {mid }}$ & $\phi_{\text {orb }}$ & \multicolumn{1}{c}{ Si IV 4089 } & \multicolumn{1}{c}{ N III 4097 } & H $\delta$ 4102 & \multicolumn{1}{c}{ Si IV 4116 } & Si/N ensemble \\
\hline b8001 & 2450006.8514 & 0.9502 & $-5.99 \pm 0.82$ & $-9.60 \pm 1.47$ & $-5.71 \pm 0.77$ & $-3.87 \pm 1.05$ & $-6.27 \pm 0.51$ \\
b8002 & 2450006.8660 & 0.9518 & $-5.31 \pm 0.83$ & $-8.05 \pm 1.43$ & $-6.52 \pm 1.06$ & $-3.89 \pm 1.04$ & $-5.63 \pm 0.51$ \\
b8004 & 2450008.8590 & 0.1741 & $23.11 \pm 0.97$ & $21.90 \pm 1.75$ & $29.18 \pm 0.81$ & $22.66 \pm 1.26$ & $22.69 \pm 0.58$ \\
b8005 & 2450009.8532 & 0.2850 & $18.14 \pm 0.71$ & $14.68 \pm 1.36$ & $24.07 \pm 1.15$ & $15.72 \pm 1.04$ & $16.79 \pm 0.45$ \\
b8006 & 2450010.8543 & 0.3967 & $10.76 \pm 0.43$ & $10.87 \pm 0.81$ & $11.75 \pm 0.94$ & $9.90 \pm 0.62$ & $10.23 \pm 0.31$ \\
\hline
\end{tabular}

Table A.2. Observing log and radial velocities of the red spectra (central wavelength $5965 \AA$ ). Columns as in Table A.1.

\begin{tabular}{lllrrrrr}
\hline Ident & BJD $_{\text {mid }}$ & $\phi_{\text {orb }}$ & \multicolumn{1}{c}{ N II 5676/9 } & \multicolumn{1}{c}{ N II 5697 } & \multicolumn{1}{c}{ Al III 5686 } & \multicolumn{1}{c}{ C III 5705 } & N/Al ensemble \\
\hline r8002r & 2450006.8545 & 0.9505 & $-10.55 \pm 1.83$ & $-7.98 \pm 3.97$ & $-11.61 \pm 2.62$ & $-2.47 \pm 4.25$ & $-10.48 \pm 1.38$ \\
r8001r & 2450012.8401 & 0.6182 & $-8.96 \pm 1.82$ & $-1.77 \pm 3.46$ & $-4.75 \pm 2.45$ & $-1.56 \pm 3.78$ & $-7.65 \pm 1.29$ \\
r8003r & 2450013.8483 & 0.7307 & $-19.48 \pm 1.88$ & $-20.12 \pm 3.48$ & $-18.55 \pm 2.93$ & $-11.60 \pm 4.74$ & $-19.25 \pm 1.38$ \\
r8004r & 2450013.8629 & 0.7323 & $-17.66 \pm 1.72$ & $-17.13 \pm 2.95$ & $-21.05 \pm 2.39$ & $-9.17 \pm 3.32$ & $-17.92 \pm 1.22$ \\
r8005r & 2450014.8585 & 0.8433 & $-7.36 \pm 1.59$ & $-10.61 \pm 2.69$ & $-7.93 \pm 1.79$ & $-1.39 \pm 3.77$ & $-7.43 \pm 1.16$ \\
\hline
\end{tabular}

Table A.3. IUE observations of HD 77581.

\begin{tabular}{clllr}
\hline $\begin{array}{l}\text { SWP } \\
\text { number }\end{array}$ & $\begin{array}{l}\text { JD mid. exp. } \\
-2440000\end{array}$ & $\begin{array}{l}\text { Exp. time } \\
(\mathrm{min})\end{array}$ & $\begin{array}{l}\text { Orbital } \\
\text { phase }^{\mathrm{a}}\end{array}$ & $\begin{array}{l}\text { Velocity } \\
\left(\mathrm{km} \mathrm{s}^{\mathrm{b}, \mathrm{c}}\right)\end{array}$ \\
\hline 1442 & 3628.708 & 180 & 0.451 & $0.0 \pm 1.5$ \\
1488 & 3634.118 & 150 & 0.055 & $5.1 \pm 1.9$ \\
2087 & 3712.994 & 125 & 0.854 & $-28.6 \pm 2.0$ \\
2390 & 3745.203 & 108 & 0.447 & $3.9 \pm 2.8$ \\
3499 & 3843.592 & 120 & 0.422 & $7.9 \pm 2.7$ \\
\hline
\end{tabular}

${ }^{a}$ Using the ephemeris of Bildsten et al. (1997) (see Table 4).

b Relative to SWP 1442; corrected for shift of interstellar lines.

${ }^{\mathrm{c}}$ Quoted are $1 \sigma$ errors.

\section{Appendix A: Log of observations}

The tables in this Appendix listing the log of observations of the blue CES spectra (Table A.1), the red CES spectra (Table A.2), and the IUE spectra (Table A.3) are only available in electronic form at the CDS via anonymous ftp to cdsarc.u-strasbg.fr $(130.79 .128 .5)$ or via http://cdsweb.u-strasbg.fr/cgi-bin/qcat?J/A+A/377/925. To provide an impression of the information contained in these tables, only the first five lines of each table are displayed here.

\section{References}

Aerts, C., De Pauw, M., \& Waelkens, C. 1992, A\&A, 286, 109 Balona, L. 1986, MNRAS, 219, 111

Bildsten, L., Chakrabarty, D., Chiu, J., et al. 1997, ApJS, 113, 367

Blondin, J. M., Kallman, T. R., Fryxell, B. A., \& Taam, R. E. 1990, ApJ, 356, 591
Evans, N. R. 1988, IUE NASA Newslett., 17, 53

Feldmeyer, A., Anzer, U., Börner, \& Nagase, F. 1996, A\&A, 311,793

Hatchett, S., \& McCray, R. 1977, ApJ, 211, 552

Heiselberg, H., \& Pandharipande, V. 2000, Ann. Rev. Nucl. \& Part. Sci., 50, 481

Horne, K. 1986, PASP, 98, 609

Joss, P. C., \& Rappaport, S. A. 1984, ARA\&A, 22, 537

Kaper, L. 2001, in Proc. The influence of binaries on stellar population studies, ed. D. Vanbeveren, \& W. van Rensbergen (Kluwer Acad. Pub.), in press

Kaper, L., Hammerschlag-Hensberge, G., \& Van Loon, J. Th. 1993, A\&A, 279, 485

Kaper, L., Hammerschlag-Hensberge, G., \& Zuiderwijk, E. J. 1994, A\&A, 289, 846

Lattimer, J. M., \& Prakash, M. 2000, Phys. Rep., 333, 121

McClintock, J. E., Rappaport, S., Joss, P. C., et al. 1976, ApJ, 206, L99

Orosz, J. A., \& Kuulkers, E. 1999, MNRAS, 305, 1320 
Srinivasan, G. 2001, in Proc. ESO workshop on Black Holes in Binaries and Galactic Nuclei, ed. L. Kaper, \& E. P. J. Van den Heuvel \& Woudt (Springer, Verlag), 45

Stickland, D., Lloyd, C., \& Radzium-Woodham, A. 1997, MNRAS, 286, L21

Telting, J. H., \& Schrijvers, C. 1997, A\&A, 317, 723

Timmes, F. X., Woosley, S. E., \& Weaver, T. A. 1996, ApJ, 457,834

Thorsett, S. E., \& Chakrabarthy, D. 1999, ApJ, 512, 288

Van Kerkwijk, M. H. 1993, Ph.D. Thesis, University of Amsterdam

Van Kerkwijk, M. H., Van Paradijs, J., \& Zuiderwijk, E. J. 1995a, A\&A, 303, 497

Van Kerkwijk, M. H., Van Paradijs, J., Zuiderwijk, E. J., Hammerschlag-Hensberge, G., \& Kaper, L. 1995b, A\&A, 303, 483 (Paper II)
Van Paradijs, J. A., Hammerschlag-Hensberge, G., Van den Heuvel, E. P. J., et al. 1976, Nature, 259, 547

Van Paradijs, J., Zuiderwijk, E. J., Takens, R. J., et al. 1977a, A\&AS, 30, 195 (Paper I)

Van Paradijs, J., Takens, R., \& Zuiderwijk, E. 1977b, A\&A, 57,221

Vogt, S. S., \& Penrod, G. D. 1983, ApJ, 275, 661

Witte, M. G., \& Savonije, G. J. 1999, A\&A, 350, 129

Zhang, W., Strohmayer, T. E., \& Swank, J. H. 1997, ApJ, 482, L167

Zuiderwijk, E. J. 1995, A\&A, 299, 79 